\title{
1 The Hoja loca1 Mutant and AUX/IAA Function in Phytomer
}

\section{Development}

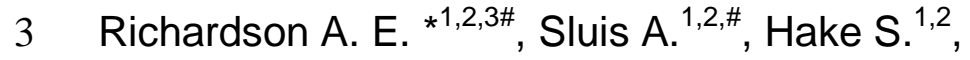

4 (1) USDA Plant Gene Expression Center, 800 Buchanan Street, Albany, CA 94710.

5 (2) Department of Plant and Microbial Biology, University of California Berkeley, CA.

6 (3) \$Institute of Molecular Plant Science, School of Biological Sciences, The University

7 of Edinburgh, Daniel Rutherford Building, Max Born Crescent, Kings Buildings,

8 Edinburgh, EH9 3BF

$9 \quad$ \# these authors contributed equally to the article.

$10{ }^{*}$ corresponding author

$11 \S$ current address

13 Short Title: Hoja loca1 and AUX/IAA Function

\section{Abstract}

16 Plant architecture is determined by the iterative building of phytomer units, each

17 containing a portion of stem, an organ and an axillary meristem. Each phytomer can

18 follow different developmental paths underpinning the complexity, and plasticity, of plant

19 form. Auxin plays a central role in the coordination of phytomer development, regulating

20 organ initiation and patterning across all axes. This diversity in auxin function results

21 from changes in the activities and expression of auxin signaling components, including

22 the AUX/IAA repressors. Higher land plants have multigene AUX/IAA families, which

23 leads to functional redundancy. Dominant mutations, which prevent AUX/IAA

24 degradation in response to auxin, have highlighted the importance of these proteins in

25 fine-tuning organ development. Here we report a new dominant AUX/IAA mutant in 26 maize, Hoja loca1 (Oja). Oja has a mutation in the degron motif of ZmIAA28 and 27 specifically affects aerial organ initiation and leaf medio-lateral patterning, but phytomer

28 initiation remains unchanged. Mutant phenotypes are variable and transcriptional

29 profiling of individual meristems identified clusters of genes that may underpin the 30 phenotypic differences. The unique phenotype of Oja provides evidence of species- 
31 specific sub-functionalization of the AUX/IAAs, and illustrates the crucial role of auxin

32 signaling in the tight coordination of phytomer unit development.

\section{Introduction}

35 The iterative post-embryonic development of plants has led to impressive

36 developmental plasticity, which allows plants to modify their architecture over time and

37 respond to environmental conditions. Organs initiate from meristems, groups of self-

38 organizing totipotent cells, in regular phyllotactic patterns. Initiation of each leaf includes

39 an axillary meristem and an internode. This developmental unit of leaf, axillary

40 meristem, and internode is known as a phytomer. Aerial plant development can

41 therefore be understood as the successive formation of phytomer units (Galinat, 1959),

42 with developmental plasticity manifesting in different amounts of growth in the three

43 components.

45 The fate of cells within the meristem is dependent on position. Cells in the center of

46 shoot meristems divide slowly, are isodiametric in shape, and are considered

47 indeterminate. As divisions push cells from the center outward, cells enter the peripheral

48 or morphogenetic zone, where organs initiate. It is here that the primordium is specified,

49 forming a leaf at plastochron $0\left(\mathrm{P}_{0}\right)$ (Bolduc et al., 2012a). The KNOTTED1-LIKE

50 HOMEOBOX (KNOX) genes mark indeterminate cell fates (Jackson et al., 1994; Smith

51 et al., 1992), and are down-regulated in $\mathrm{P}_{0}$ cells. KNOX gene expression is maintained

52 within the other components of the phytomer, the internode and bud, until they elongate

53 (Tsuda et al., 2017). The downregulation of KNOX genes in the $\mathrm{P}_{0}$ is preceded by the

54 formation of an auxin maximum, coordinated by a convergence point of the auxin

55 transporter PINFORMED1 (PIN1) in the epidermis (Bayer et al., 2009; Benková et al.,

56 2003; O'Connor et al., 2014; Reinhardt et al., 2003). Auxin acts by triggering the

57 degradation of AUX/IAA proteins, allowing AUXIN RESPONSE FACTORS (ARFs) to

58 regulate downstream genes (Lavy and Estelle, 2016; Salehin et al., 2015). These

59 coordinated events comprise the mechanism for organ initiation. 
61 As organs develop and differentiate, asymmetries are established that determine tissue 62 identity across proximal/distal, abaxial/adaxial, and medial/lateral axes (Kuhlemeier and 63 Timmermans, 2016; Richardson and Hake, 2019). In grasses, the leaf initiates as a 64 ring-shaped primordium around the meristem with the separation of the lower leaf 65 (sheath) margins occurring later in development (Johnston et al., 2015; Sharman, 66 1942). Proximal-distal patterning in the grass leaf leads to the formation of a distal blade 67 and a proximal sheath. The ligule and auricle form at the boundary of blade and sheath. 68 For most grasses, the medial/lateral axes are marked by a midvein at the center which 69 is thickened in comparison with the margins of the leaf (referred to as the midrib).

71 Auxin signaling has been implicated in all stages and axes of grass leaf development.

72 Treatment of maize shoot apices, with the auxin inhibitor NPA ( $N$-1-Naphthylphthalamic 73 Acid) limits organ initiation and prevents the separation of the sheath margins resulting 74 in a tube leaf (Scanlon, 2003). PIN proteins are also expressed at the position of a ligule 75 prior to its elaboration (Moon et al., 2013) and RNAseq analysis of leaf microdomains 76 has shown that ligule development involves the recapitulation of the organ initiation 77 mechanism (Johnston et al., 2014). The establishment of the abaxial/adaxial axis also 78 involves auxin signaling, utilizing opposing gradients of tasiR-ARFs and miR166 79 (Nogueira et al., 2007). Despite the role of auxin signaling throughout grass leaf 80 development, auxin signaling mutants (i.e. mutants in AUX/IAA or ARFs), which affect 81 leaf development, have not yet been reported in grasses.

83 Here we describe a dominant mutant, named Hoja loca1 (Oja), with a range of leaf 84 defects. In its most severe expression, mutants fail to initiate leaves, although an 85 internode will be partitioned to each phytomer. In the least severe, midveins are missing 86 from otherwise correctly patterned leaves. Between these extremes, tube leaves are 87 formed, where the leaf margin is completely fused. Remarkably, leaves maintain 88 proximal distal and abaxial/adaxial patterning. Using positional cloning and whole 89 genome sequencing, we identify the gene as the AUX/IAA protein, ZmIAA28 90 (GRMZM2G035465). By analysing individual meristems, we were able to use the 91 variable phytomer phenotypes of Oja to dissect potential regulatory roles of ZmIAA28 
92 that may be responsive to different auxin concentrations. Unlike other maize AUX/IAA

93 mutants that specifically affect roots (Woll et al., 2005) or inflorescences (Galli et al.,

94 2015), Oja provides a view into the unique role of AUX/IAA proteins in grass leaf 95 patterning. The ability of Oja to produce a leafless phytomer also hints at the role of 96 auxin signaling in coordinating development of the separate phytomer components.

\section{Results}

99 Hoja loca1

100 Hoja loca1 (Oja) is a dominant mutant that was discovered in a forward genetics EMS 101 screen. The phenotype is variable, ranging from mild to severe, and it exhibits 102 unpredictable expression through generations; crosses made with mildly affected plants give rise to severe plants and vice versa.

Hoja loca1 leaves have medial/lateral defects but normal proximal/distal and abaxial/adaxial patterning in aerial organs

108 a distal blade that bends away from the plant, optimized for photosynthesis (Figure 1Ai).

109 At the junction of the blade and sheath is the ligule, an epidermal fringe on the adaxial

110 surface and two auricles along the margin that allow the blade to tilt away from the 111 sheath (Figure 1Aii-iv). The blade contains a midrib, a thickened region with chlorophyll112 containing cells on the abaxial surface and clear cells on the adaxial surface (Figure $1131 \mathrm{Av}$ ), that supports the extended blade.

114 In contrast to wild-type seedlings, Hoja loca1 (Oja) mutants have varied leaf 115 phenotypes present on the same plant, including normal leaves (Figure 1B,C). The 116 mildest leaf mutant phenotype has missing midribs (leaf 2 in Figure 1Bi). These leaves 117 droop backwards without the structural support of the midrib (Figure 1Bi-v). The 118 midribless leaves completely lack adaxial clear cells, although the flanking blade 119 regions appear normal (Figure 1Bv). Midribless leaves still have normal ligules and 120 auricles (Figure 1Bii-iv, arrowheads) suggesting that the midrib is not essential in 121 elaboration of proximal/distal patterning. 
Severe mutant leaf phenotypes include tube leaves in which the margins are not

123 separated (Figure 1C). As in the case for midribless leaves, tube leaves also have

124 normal proximal/distal patterning, developing a normal ligule between the sheath and

125 blade (Figure Cii-iv). Cells in the tube leaves also have correct abaxial/adaxial polarity

126 with chlorophyll containing cells on the abaxial surface and clear cells on the adaxial

127 surface (Figure 1Cv). These results suggest that Oja does not influence proximal/distal

128 or abaxial/adaxial patterning.

129 To determine whether there was a pattern in initiation of different leaf

130 phenotypes, we recorded the occurrence of midribless and tube leaves in a population

131 of 150 plants (Figure 1D). Both midribless and tube leaves can be observed in the same

132 plant. In all cases, the first leaf is never affected. The second leaf may show one of

133 these defects and the third and fourth are most likely to be mutant (Figure 1D). The

134 frequency for both defects decreases during development but tube leaves could be

135 observed late in development. This variability of phytomer phenotype makes Oja difficult

136 to work with, but also provides a unique opportunity to dissect the different functions of

137 the underlying gene.

138 In many Oja plants, inflorescence branching and floral development was severely

139 affected. Both male and female inflorescences bore bare regions indicative of a failure

140 to initiate spikelet primordia, and the tassels were not branched. However, when flowers

141 did form, they appeared to be normal and fertile (Supplementary Figure 1). In contrast

142 to aerial development, root development in seedlings appeared to be normal with

143 initiation of primary, seminal and lateral roots and a normal gravitropic response

144 (Supplementary Figure 2). Oja therefore specifically affects aerial organ development.

146 Hoja loca1 leaves have defects in vein distribution, but normal vein density.

147 Given the midrib defect, we next questioned whether veins are altered in Oja mutants.

148 Seedling leaf cross-sections showed that the abaxial/adaxial patterning of vascular

149 bundles appears normal (Figure 2A,B), mirroring the normal abaxial/adaxial patterning

150 of the entire organ.

151 To assess whether the medio-lateral patterning of vascular bundles was altered 152 we measured leaf width and determined vein density and distribution in the third leaf of 
153 3-week-old seedlings. Midribless leaves were narrower than wild type and tube leaves 154 were less than half the width of wild type (Figure 2C). Overall vein density was not 155 affected by Oja (Figure 2D) suggesting a reduction in total vein number to match the 156 decrease in size. To assess vein distribution, we counted the number of intermediate 157 veins between lateral veins in both wild-type and midribless leaves. In wild type, the 158 number of intermediate veins shows a symmetry across the leaf, with a dip at the mid 159 position (Figure 2Eii, blue). We did not identify a dip of intermediate veins in the midvein 160 position in Oja midribless leaves (Figure 2Eii, orange), supporting the hypothesis that 161 medio-lateral patterning is affected in the Oja mutant.

\section{Hoja loca1 mutants fail to initiate leaves but still set aside internode cells}

164 At each leaf initiation, cells are set aside for the leaf and the internode below (Johri and 165 Coe, 1983). A mature maize plant is thus composed of alternating internodes and 166 nodes, which is the position of leaf insertion. In mature Oja plants we observed bare 167 nodes, for example the plant in Figure 3A ended in a tube leaf after four nodes failed to 168 elaborate leaves (arrows). To assess whether these bare nodes were due to ectopic 169 nodes or a failure of leaf initiation, we counted the number of nodes in Oja versus wild170 type siblings and compared these to the number of leaves. In mature plants there is no 171 significant difference in number of nodes (Figure 3B), but there are significantly fewer 172 leaves in Oja mutants (Figure 3C). This alternating node, internode, node pattern 173 without accompanying leaves is also observed when slicing through developing tube 174 leaves (Figure 3D). These data suggest that Oja may be able to initiate new phytomers 175 consisting of just nodes and internodes.

176 To identify whether leaves failed to initiate, or whether they initiated but failed to 177 elaborate, we used scanning electron microscopy (SEM) to examine the earliest 178 primordial stages of leaf development. Wild-type maize leaves at the $\mathrm{P}_{1}$ stage are a ring 179 of cells that surround the meristem. At $\mathrm{P}_{2}$, the leaf begins to grow in the proximal distal 180 dimension, with growth highest at the midvein position. By $P_{3}$, the leaf primordium 181 covers the top of the meristem and the margins begin to expand (Figure 3E). Further 182 growth of the leaf margins at the $\mathrm{P}_{4}$ stage envelopes the younger leaves and meristem 183 (Hay and Hake, 2004; Scanlon et al., 1996). Longitudinal growth of the internode and 
184 sheath occur later in development. Compared to wild-type seedlings where developing

185 leaf primordia always obscured the internodes, the internodes were often visible in 186 severe Oja shoots (Figure 3F-I). The meristem in Figure 3F has a single ring 187 primordium which may be the early stages of a tube leaf, and below this ring, no older 188 leaves have initiated, leaving an elongated, exposed internode. The meristem in Figure 189 3G initiated five internodes without leaves, before more leaf primordia were initiated. In 190 some Oja mutants, the leaf initiation defect was so severe that the shoot apex ended in 191 pin-like barren meristems as in Figure 3H. In many mutants, axillary meristems formed 192 which contained more normal leaf initiation (Figure 3I). In fact, the mature plant in 193 Figure 3A had a fertile tiller although the main shoot ended in a tube leaf with skipped 194 leaf initiation. The SEM data suggest that the discrepancy between node number and 195 leaf number in Oja is due to patterning during the earliest stages of leaf primordium 196 initiation. This finding supports the hypothesis that Oja mutants fail to initiate leaves, 197 despite initiating new phytomers.

198 The skipped leaf initiation also explains some of the seedling architecture 199 differences. For example, the normal seedling in Figure $1 \mathrm{~A}$ has four visible leaves, while 200 the seedling in Figure 1B has only three leaves visible, with the second leaf the size of a 201 normal third leaf. In addition, the distance between the first and second leaf is much 202 greater than in the wild type. This observation suggests that leaf two was skipped in the 203 mutant.

Hoja loca1 encodes the AUX/IAA protein, ZmIAA28.

206 To identify the causal mutation, we mapped the Mo17 Oja region in a backcross 207 population to A619. Bulk segregant mapping placed Oja on chromosome 8 (Figure 4).

208 SSR primers were used to narrow the position to an interval of $1 \mathrm{Mb}$ containing 19 209 annotated genes (Figure 4Aiii). Using published RNAseq data we narrowed the 210 candidate gene list further, as only 10 genes in the interval were reported as expressed 211 in the shoot apical meristem or leaf primordia (Figure 4Aiii, green).

212 To further map the interval, we used whole genome sequencing of two bulk 213 pools, one wild type and one mutant pool, each with 40 individuals. A large interval was 214 detected on chromosome 8 between 75 and 135 Mb. To generate a candidate SNP list, 
215 we removed SNPs reported in the HapMapv3 from Panzea (www.panzea.org/data) and

216 limited the reported SNPs to EMS-type $\mathrm{G}$ to $\mathrm{A}$, or $\mathrm{C}$ to $\mathrm{T}$. Within the large interval, there

217 were nine moderate or high-effect SNPs within annotated gene models (Figure 4Ai), but

218 only one SNP detected within the fine SSR mapping interval (Figure 4Aiii, * ). This

219 change was a C to $\mathrm{T}$ nucleotide transition in GRMZM2G035465, which encodes

220 ZmIAA28 (Figure 4B). The $\mathrm{C}$ to $\mathrm{T}$ transition causes a missense mutation from a Glycine

221 to Glutamic Acid in the center of the highly conserved degron motif (Figure 4C). Similar

222 mutations in other AUX/IAA proteins prevent degradation in response to high auxin

223 (Reed, 2001) suggesting that Oja is caused by a stabilizing mutation in the degron

224 motif, preventing degradation of ZmIAA28 in the presence of auxin.

Hoja loca1 shows dysregulation of auxin signaling.

227 To assess the effect of the Oja1 mutation on gene expression in vegetative meristem

228 and developing leaf primordia, we sequenced mRNA extracted from dissected 229 meristems with P1/P2 primordia attached from 3-week-old seedlings (Figure 5A). Given

230 the variability in phenotype, each individual was used to make a single library, with 36

231 libraries sequenced in total. By scoring the libraries based on the seedling leaf 232 phenotype, we classified the libraries into two groups: wild type and mutant (orange and 233 blue annotations indicated on the principal component analysis plot in Figure 5B).

234 Differential gene expression analysis identified 2877 significantly differentially 235 expressed genes (padj<0.05) with a log2FC of $>0.59$ or $<0.59$, of which 689 were down 236 in the mutant-scored samples compared to the wild type. GO term analysis showed 237 enrichment for many terms, including those associated with auxin signaling, meristem 238 regulation and leaf development (Figure 5C). Consistent with the phenotype of Oja, 239 genes associated with leaf development are down, and those associated with meristem 240 regulation are up in the mutant-scored samples compared to the wild type-scored 241 samples. This suggests that ZmIAA28 has a role in regulation of auxin response, and 242 leaf development. 
244 Transcriptomes of individual Hoja loca1 meristems and leaf primordia can 245 differentiate four distinct transcriptional profiles, correlated with the four 246 phytomer phenotypes.

247 To further investigate how the different leaf phenotypes of Oja may arise, we 248 interrogated the transcriptional profiles of each individual sample. We asked whether we 249 could identify four transcriptional profiles representing the four potential phenotypes of 250 an initiating Oja phytomer: normal leaf, midribless leaf, tube leaf and no leaf initiation.

251 We first filtered the expressed gene list to those shown to be expressed in the 252 meristem and developing leaf primordia based on (Knaeur 2019) to reduce noise 253 caused by differing amounts of stem and internode tissue harvested with each sample. 254 Using the top 1500 most variable of these genes, we carried out hierarchical clustering 255 and identified four clusters using K-means (Figure 6A). Overlaying these clusters on a 256 PCA plot of the top 100 most variable of all expressed genes showed clear separation 257 of the four groups along PC1 (Figure 6B). This indicated that the four clusters (C1-4) 258 were transcriptionally distinct.

259 To assess which cluster may be associated with each phytomer phenotype, we 260 generated self-organizing maps (SOMs) of all expressed genes across C1-4 (Figure 261 6D) and annotated the nodes with genes involved in leaf initiation and development. A 262 defining feature of initiating leaf primordia is the specific downregulation of KNOX genes 263 in the $\mathrm{P}_{0}$ and the activation of leaf patterning genes. For the four phytomer phenotypes; 264 normal leaf, midribless leaf, tube leaf and no leaf initiation; we predicted that these 265 genes would show distinct profiles. In phytomers initiating normal leaves we would 266 expect low KNOX gene expression, with high leaf patterning gene expression, and the 267 converse for phytomers failing to initiate leaves. Phytomers initiating midribless leaves 268 and tubes leaves would have an intermediate profile. We found that most KNOX genes 269 were in node 14, (Figure 6D) where expression was at the lowest level in C2 (Figure 270 6E), consistent with KNOX genes being downregulated during normal leaf initiation.

271 Genes associated with leaf development were found in nodes where expression was 272 highest in C2 (e.g. node 7 contains CUP-SHAPED-COTYLEDON genes and 273 WUSCHEL-LIKE-HOMEOBOX genes known to be involved in leaf development and 
274 organ initiation). This supports the hypothesis that C2 represents meristems initiating 275 phytomers with normal leaves.

276 Conversely, higher KNOX (node 14) and lower leaf development gene 277 expression (e.g. nodes 7 and 8) associated with C4 (Figure 6D). This is consistent with 278 the hypothesis that C4 represents phytomers with a 'no leaf initiation' phenotype. This is 279 further supported by the expectation that phytomers failing to initiate leaves will be most 280 transcriptionally distinct from those initiating normal leaves, and the PCA and 281 hierarchical clustering both show C4 as most different to C2 (Figure 6B). Combined, 282 these data suggests that C2 represents phytomers initiating normal leaves, and C4 283 represents phytomers with no leaf initiation.

284 To identify which of the remaining two clusters may correlate with either the tube 285 leaf or midribless leaf phytomer phenotype we analysed the expression profiles of 286 medio-lateral leaf patterning genes. We predicted that these genes would be 287 misregulated in phytomers initiating midribless or tube leaves, and be downregulated in $288 \quad$ C4 as these phytomers are not initiating leaves.

289 Tube leaves lack leaf margins, and this should be reflected by reduced 290 expression of genes like the WOX gene NARROWSHEATH1 (NS1) which defines the 291 leaf margin (Nardmann et al., 2004; Scanlon and Freeling, 1997). Many WOX genes, 292 including NS1, are found in node 7 where expression is lowest in C3 and C4. Low WOX 293 expression in C3 could indicate that this group represents phytomers initiating tube294 shaped leaves.

295 Few genes have been identified as responsible for midribless identity, but the 296 YABBY genes DROPPING LEAF 1 (DRL1/IFA1) and 2 (DRL2)(Strable et al., 2017) 297 both have defects in midrib development. We would predict that genes involved in 298 midrib development would be downregulated in phytomers initiating midribless leaves. $299 D R L 1$ and $D R L 2$ are located in node 8, where expression is highest in C2 and low in all 300 other clusters, making it difficult to differentiate between C1 and C3. Given that the 301 midribless leaf phenotype appears most similar to a normal leaf, we predict that the 302 cluster most similar to C2 would represent phytomers initiating midribless leaves. Based 303 on the PCA and the differential gene expression analysis, C1 was most similar in 304 transcriptional profile to $\mathrm{C} 2$ (Figure 6B,C, pink). We therefore hypothesise that $\mathrm{C} 1$ 
305 represents phytomers initiating midribless leaves, and C3 represents phytomers 306 initiating tube leaves.

Each phytomer phenotype can be associated with specific gene expression changes.

310 To further test the hypothesis that each cluster represents a different phytomer 311 phenotype (C1- midribless leaf, C2- normal leaf, C3- tube leaf, C4- no leaf initiation), we 312 carried out differential gene expression analysis for each 'mutant' cluster (C1, 3 and 4) 313 in comparison with the hypothesised normal leaf representative (C2). Overlap of the 314 significantly differentially expressed gene lists (padj $<0.05$, log2FC $<-0.59$ or $>0.59$, 315 Figure 6C, E-H) showed distinct transcriptional profiles for each cluster, suggesting that 316 each phytomer phenotype may be underpinned by different gene expression changes 317 triggered by variable effects of ZmIAA28 stabilization on auxin signaling.

318 To identify genes potentially important for each phenotype we overlaid the 319 significantly differentially expressed genes (log2 fold change $>0.59$ or <-0.59) for each 320 comparison, and identified 170 genes specific to C1, 601 to C3, and 2288 to C4. C4 321 showed the most unique significantly differentially expressed genes, many of which are 322 important components of leaf initiation. These genes included the boundary genes 323 CUC2 and CUC3, which were significantly downregulated, consistent with their roles in 324 specification of the boundary between the primordium and meristem (Hibara et al., 325 2006; Aida et al., 1999). This further supports the hypothesis that C4 represents 326 phytomers which fail to initiate leaves, and suggests ZmIAA28 degradation in the 327 presence of auxin is involved in triggering leaf initiation.

328 We predict that C1 and C3 represent phytomers initiating leaves with medial329 lateral patterning defects. WOX genes exhibited distinct gene expression profiles across 330 the clusters, with opposite expression profiles in C3 compared to C2. WOX3b, 4, 5a and 33113 were significantly upregulated in C3, whereas WOX3a, 9a, 9b, and NS1 were all 332 significantly downregulated (Figure 6F). This finding suggests that medial-lateral 333 patterning and marginal domain development is severely perturbed in C3, correlating 334 with the tube leaf phenotype. The analysis demonstrates that auxin signalling via 
335 ZmIAA28 degradation is important in regulation of WOX gene expression for medial336 lateral domain patterning.

$337 \quad$ Midribless leaves show defects in midrib formation and in vein distribution in the 338 leaf blade. We therefore analysed the expression profiles of genes with potential roles in 339 midrib and vein patterning. YABBY1, 9, 15, DRL1 and DRL2 are all significantly 340 downregulated in C1 compared to C2 (Figure 6G). This finding suggests that ZmIAA28 341 may link auxin signalling with midrib formation via regulation of DRL1 and 2. C1 also 342 showed significant downregulation of multiple SHORT INTERNODES (SHI) / STYLISH 343 (STY) / SHI RELATED SEQUENCE (SRS) genes (Figure 6H), which in Arabidopsis are 344 involved in vascular patterning (Baylis et al., 2013) and have been shown to be 345 regulated by AUX/IAA proteins (Singh et al., 2020). ZmIAA28 may therefore also be 346 involved in auxin regulation of SRS gene expression during grass leaf vascular 347 patterning.

ZmIAA28 is involved in the auxin maxima maintenance preceding leaf initiation

350 The no leaf initiation phenotype of Oja led us to ask if we could use Oja to dissect the 351 link between organ initiation and auxin maxima formation. Using the comparison 352 between $\mathrm{C} 4$ and $\mathrm{C} 2$, we first interrogated the expression profile of genes known to be 353 involved in organ initiation. We then used in situ hybridisation and immunolocalisation to 354 test gene expression in a spatial manner.

355 In C4, KNOX genes are significantly upregulated, indicating that KNOX gene 356 downregulation is aberrant in Oja meristems in which leaf initiation fails. We used 357 antibodies to KNOTTED1 (KN1) (Smith et al., 1992) in order to follow leaf initiation 358 defects in the meristem. KN1 accumulates in the meristem and unexpanded stem and is 359 clearly down-regulated at the $\mathrm{P}_{0}$ region, remaining absent in normal maize leaves 360 (Figure 7A). In Oja mutants we see strong accumulation at the tip of the meristem but, 361 unlike the normal meristem, KN1 down-regulation appears on both sides of the 362 meristem, suggesting that this $P_{0}$ encircles the meristem (Figure 7B). The encircling 363 downregulation could be indicative of tube leaf initiation. This particular meristem has 364 also failed to initiate at least two leaves, and KN1 localisation is still maintained in the 365 internode region below the meristem (Figure 7B). This variable downregulation of KNOX 
366 genes supports the hypothesis that ZmIAA28 may be upstream of the downregulation of KNOX genes in initiating primordia. in the epidermis to the site of leaf initiation, where an auxin maxima forms. From the $\mathrm{P}_{0}$, 370 PIN1 proteins move auxin toward pre-existing vasculature, predicting the path of 371 provascular traces (Reinhardt et al., 2003). In many species outside of the Brassica, 372 PIN1 proteins have duplicated and diversified function. Sister of PIN1 (SoPIN1) 373 localizes at the leaf initiation site and is also detected in vasculature in grasses 374 (O'Connor et al., 2014). PIN1a and PIN1b have roles in vascular formation and stem 375 growth (O'Connor et al., 2017). We found that all PIN gene expression was significantly 376 downregulated in C4 compared to $\mathrm{C} 2$, suggesting that the feedback mechanism that 377 maintains auxin maxima during organ initiation may be defective in Oja. As SoPIN1 is 378 essential for organ initiation in the grasses (O'Connor et al., 2017) and is redundantly 379 involved in vascular development, we chose to focus on SoPIN1 accumulation in the 380 plant. To determine whether SoPIN1 patterning was defective in Oja meristems, we 381 used an antibody to SoPIN1 to detect protein accumulation at the $\mathrm{P}_{0}$ site and in the 382 developing pre-vascular strand in wild-type plants (Figure 7C). In wild-type meristems, 383 SoPIN1 convergence points form in the epidermis of the meristem at the $P_{0}$, which 384 persists through to the tip of the $\mathrm{P}_{1}$ leaf (Figure $7 \mathrm{C}$ ). Strong internal traces of SoPIN1 385 are also observed highlighting the developing vascular strands that link the primordium 386 apex to the stem vascular system (Figure 7C). In the Oja mutant shown, which has a 387 number of skipped leaf initiations and appears as a pin, the SoPIN1 localization is 388 throughout the tip of the meristem rather than localizing strongly to the $\mathrm{P}_{0}$ region (Figure 389 7D). Normal accumulation and polarization can be seen in a vein trace within the mutant 390 meristem, but this does not connect to the epidermis. This result suggests that SoPIN1 391 can polarize and be coordinated across the tissue. However, SoPIN1 fails to form a 392 strong convergence point needed at the $\mathrm{P}_{0}$ in Oja, implying that ZmIAA28 may be 393 involved in the feedback regulation of SoPIN1 at lateral organ initiation sites, essential 394 for organ initiation. In support of this hypothesis we observe changes in the expression 395 of several genes involved in PIN protein localisation, including the PINOID protein BIF2 396 (McSteen and Hake, 2001), which is significantly downregulated in C4 compared to C2. 
Downstream of auxin maxima formation and KNOX gene downregulation is activation of leaf specific genes, such as DRL1 and 2 (Strable et al., 2017). DRL genes are early markers of leaf patterning and are expressed at the $\mathrm{P}_{0}$ and at the midrib in leaf primordia. To explore the effect of Oja on DRL expression, we used in situ hybridization. In the normal seedling, expression was detected in the $P_{0}$ and each subsequent midvein region (Figure 7E). In the Oja mutant shown, DRL expression was similar at the midvein region in normal developing leaves, however, there was no detectable expression in the $P_{0}$ and fewer leaves had initiated. As DRL is involved in midrib specification (Strable et al., 2017), this lack of DRL in the $\mathrm{P}_{0}$ could be due to the lack of leaf initiation or due to the formation of a midribless leaf. Nevertheless, as an early marker of leaf patterning this expression pattern supports the hypothesis that Oja mutants fail to specify the $\mathrm{P}_{0}$ leaf correctly despite defining the rest of the phytomer.

In summary, these findings suggest that Oja has a defect in leaf initiation and patterning, not just leaf outgrowth, which leads to the reduced leaf number compared to node number.

\section{DISCUSSION}

The dominant Hoja loca1 (Oja) mutant, caused by a mutation in ZmIAA28, specifically affects aerial organ initiation and medial/lateral patterning in the leaf,

416 illustrating highly specific AUX/IAA function essential for the fine-tuning of plant 417 development by auxin. By analysing individual meristems, we were able to use the 418 variable phytomer phenotypes of Oja to dissect potential regulatory roles of ZmIAA28 419 that may be responsive to different auxin concentrations.

AUX/IAA proteins function as repressors in low auxin conditions by interacting 421 with ARF transcription factors and preventing their activation of auxin responsive genes.

422 An increase in auxin promotes an interaction between AUX/IAA and Transport Inhibitor 423 Response1 (TIR1), an F-box component of the SCF complex that targets proteins for 424 degradation. Binding of AUX/IAA to TIR1 in the presence of auxin leads to AUX/IAA 425 degradation and thus releases ARF repression (Dharmasiri et al., 2005; Kepinski and 426 Leyser, 2005). As the AUX/IAA gene family is large, redundancy has mostly hidden 427 loss-of-function phenotypes (Overvoorde et al., 2005). Instead, genetic analysis has 
428 identified dominant AUX/IAA mutations that stabilize these normally short-lived proteins 429 by disrupting their proteolysis or reducing their ability to bind to TIR1 (Ouellet et al., 430 2001; Ramos et al., 2001; Tian et al., 2003; Worley et al., 2000; Yang et al., 2004). Most 431 mutations, including Oja, are located in domain II in the highly conserved core degron 432 motif (Fukaki et al., 2002; Nagpal et al., 2000; Rogg et al., 2001; Rouse et al., 1998; 433 Zenser et al., 2001) which interacts with TIR1 in the presence of auxin (Tan et al., 434 2007).

435 Nine clades of AUX/IAA proteins have been identified (Matthes et al., 2019), 436 allowing for significant subfunctionalization during the evolution of the AUX/IAA family.

437 The majority of Arabidopsis AUX/IAA mutants were first described by their agravitropic 438 root, reduced lateral roots, and/or shorter root phenotypes (Fukaki et al., 2002; Nagpal 439 et al., 2000; Rouse et al., 1998). Mutants that were identified by a shoot phenotype such 440 as bodenlos, Shy2-2 and axr3 have pleiotropic phenotypes, also affecting the roots. For 441 example, the semi-dominant Shy2 mutant was identified as a suppressor of a 442 phytochrome elongated hypocotyl phenotype but also has root defects and curled 443 leaves (Reed et al., 1998; Tian and Reed, 1999). Embryonic patterning defects were 444 identified in bodenlos homozygotes but mature heterozygous plants also have root 445 defects (Hamann et al., 2002). Axr3 mutants develop curled leaves (Leyser et al., 1996; 446 Pérez-Pérez et al., 2010) due to defects in coordination between the abaxial and 447 adaxial surfaces of the leaf. When these mutant phenotypes are mapped to the 448 AUX/IAA clades, no obvious pattern is observed, suggesting that function is not 449 conserved within a clade. This finding highlights the importance of studying the specific 450 function of AUX/IAA proteins in multiple species, as genetic similarity is unlikely to 451 correspond to related functionality.

452 Our work suggests that ZmIAA28 acts specifically during aerial organ initiation, 453 potentially upstream of KNOX downregulation. This contrasts with the function of the 454 tomato class VIII AUX/IAA, SIIAA9. Tomato leaf margin complexity is dependent on the 455 ectopic expression of KNOX genes in the leaf lamina. However, reduction of SIIAA9 456 expression causes a loss of leaf margin complexity, independent of KNOX gene 457 expression as determined by RT-PCR (Wang et al., 2005). This suggests that although 458 ZmIAA28 and SIIAA9 are in the same clade, they differ in their relationship with KNOX 
459 gene regulation. This further argues for the importance for studying specific AUX/IAA 460 functions in different species.

AUX/IAA mutants in maize appear to be less pleiotropic than Arabidopsis; their defects are confined to specific parts of the plant. For example, the rootless with undetectable meristem (rum1) mutant is deficient in primary and lateral root initiation with no defects in the aerial parts (Woll et al., 2005). rum1 encodes ZmIAA10 and, unlike other described AUX/IAA mutants that have a missense mutation in the degron, rum1 carries a deletion that includes the GWPPV degron sequence in domain II (Von Behrens et al., 2011). The Bif1 (ZmIAA27) and Bif4 (ZmIAA20) AUX/IAA mutants reduce the branching in male and female inflorescences (Barazesh et al., 2009; Galli et al., 2015), however there is no effect on vegetative growth (Galli et al., 2015). Oja mutants have striking defects in leaf development but have normal roots and flowers if they make inflorescences. This suggests that ZmIAA10, ZmIAA20, ZmIAA27 and ZmIAA28 have tissue-specific functions. These specificities do not appear to arise from

473 tissue specific expression as, based on published RNAseq data, ZmIAA27 and 474 ZmIAA28 have similar expression patterns. RUM1 is expressed at very low levels 475 compared to a close paralog which is expressed at high levels in many root tissues as 476 well as the leaf (Zhang et al., 2016). Perhaps the tissue specificity results from the 477 interaction of each AUX/IAA with different ARFs or different TIR proteins and these are 478 responsible for the observed phenotypes in the dominant mutants (Salehin et al., 2015). Through investigating leaf initiation, we found that SoPIN1 failed to converge at 480 the $\mathrm{P}_{0}$ in Oja mutants suggesting that ZmIAA28 plays a role in the feedback signaling 481 required for forming the convergence point that triggers organ initiation. A role for the 482 TIR/ARF/AUX/IAA module in regulating cellular polarisation has also been observed in 483 Arabidopsis in which induction of the ARF, MONOPTEROS (MP) in the mp mutant 484 background triggered spontaneous PIN1 convergence point formation and primordium 485 outgrowth in the normally barren $m p$ meristem (Bhatia et al., 2016). Interestingly, 486 although leaf initiation was severely affected in Oja, many other organ initiation events 487 occurred normally. For example, ligule development requires reactivation of the organ 488 initiation pathway (Johnston et al., 2014) but Oja mutants have no ligule defect, 489 suggesting that different AUX/IAAs may be involved in ligule development. 
Given that formation of the SoPIN1 convergence point at the $P_{0}$ stimulates midvein development, it is likely that ZmIAA28 is required for the signaling that promotes midrib formation at the midvein, influencing medio-lateral patterning and

493 possibly subsequent $D R L$ expression. Supporting the role of auxin signaling upstream 494 of $D R L$ expression, DAPseq analyses in maize have identified ARF binding sites upstream of both maize DRL genes (Galli et al., 2018). In midribless Oja mutant leaves,

496 the midrib domain appears to be completely lost, which is more severe than in the $d r l$ 497 double mutant (Strable et al., 2017), suggesting that other factors, also influenced by 498 auxin signaling, are required to specify midrib differentiation in addition to DRL.

499 Mediolateral development in the leaf is driven by WOX gene activity (Conklin et 500 al., 2021; Zhang et al., 2020). Our transcriptional analyses highlight wide-spread miss501 regulation of WOX gene expression in Oja, with most significant downregulation 502 associated with potential tube leaf formation, and failure of leaf initiation. The formation 503 of tube-shaped leaves in maize can be induced by incubation with the auxin inhibitor 504 NPA (Scanlon, 2003) which leads to a failure of sheath margin separation, and this 505 correlates with a high accumulation of ZmPIN1a in this region at the P4 stage of leaf 506 development (Johnston et al., 2015). Recent work has also shown that NS1 is induced 507 by auxin treatment (Conklin et al., 2021). This suggests that ZmIAA28 may be involved 508 in translation of the auxin concentration signal to leaf margin specification and later 509 margin development.

$510 \quad$ Analysis of proximal-distal patterning mutants gave rise to the hypothesis that the 511 midrib domain acts as a signaling centre to coordinate ligule development from the 512 midrib to the leaf margins (Becraft and Freeling, 1991; Harper and Freeling, 1996; Moon 513 et al., 2013). However, proximal-distal patterning is normal in Oja leaves lacking the 514 midrib. This suggests that it is not the midrib domain itself that links medial-lateral and 515 proximal-distal patterning but potentially medial-lateral patterning factors that are largely 516 independent of ZmIAA28 regulation of midrib formation.

517 The variability of the Oja phenotype is not unusual, having also been observed in 518 other AUX/IAA mutants such as in the Arabidopsis mutant bodenlos, with some mutants 519 missing hypocotyl and root and others just missing a root (Hamann et al., 2002). These 520 variable phenotypes could arise from stochastic over- and under-compensation of the 
521 auxin signaling network. The sensitivity of different tissues to these stochastic changes 522 in auxin concentration could arise through different combinations of TIRs, ARFs and 523 other auxin signaling components (Powers and Strader, 2020; Parry et al., 2009;

524 Havens et al., 2012). In such a scenario, the four clusters of gene expression and 525 corresponding phytomer phenotypes identified in this work, may represent stable end526 states that arise from perturbations of the auxin pathway very early in development. As 527 such, when ZmIAA28 is not degraded in the presence of high auxin, as it is in wild type, 528 the three mutant phytomer phenotypes result from sub-optimal compensatory 529 mechanisms cascading to islands of stability. Oja therefore reveals three regions where 530 response to high auxin concentrations during the earliest phases of leaf development is 531 crucial in defining leaf shape: initiation, formation of a midrib/ vascular patterning, and 532 separation of the leaf margins.

533 The phytomer consists of a node, internode, axillary bud and organ. To maintain 534 correct plant architecture, the development of each component is tightly regulated. Oja 535 is unusual in that this coordination within the phytomer unit is apparently broken, 536 allowing the initiation of nodes and internodes without accompanying organs. This 537 finding suggests that auxin is not only involved in the initiation of organs, but also in the 538 regulation of phytomer development as a whole. The Oja mutant illustrates the species539 specific subfunctionalisation of the AUX/IAA proteins in regulating plant development. In 540 contrast to other known AUX/IAAs, ZmIAA28 specifically affects initiation of aerial 541 organs and impacts medio-lateral patterning. As further studies identify functions of 542 other grass AUX/IAAs it will be of interest to compare them to Oja to see how far this 543 subfunctionalisation holds. This will be particularly interesting to compare to Arabidopsis 544 aux/iaa mutants which appear to be more pleiotropic, and to determine the reasons for 545 the species-specific differences. 


\section{Methods}

\section{Isolation of Hoja loca1}

549 Hoja loca1 (Oja) was discovered by a forward genetic approach. Pollen of the Mo17

550 maize inbred was treated with ethyl methanesulfonate (EMS) and crossed onto the B73

551 inbred. Hybrid F1 seed were planted in the field and screened. The single mutant plant 552 was open pollinated and crossed to multiple inbreds the following season. Further 553 crosses established the dominance of the mutant phenotype although often less than $55450 \%$ of the plants showed the phenotype, indicating low phenotypic penetrance.

555 Each generation displayed unpredictable variability in phenotypic expression ranging 556 from mild to severe. Crosses made with mildly affected plants gave rise to severe plants 557 and vice versa. Due to the severity of the phenotype, homozygotes were rarely 558 obtained, thus the observations are exclusively with heterozygotes. Analysis was 559 carried out in plants with at least four backcrosses to B73.

\section{Plant Growth Conditions}

562 Analyses were carried out in families segregating for Hoja loca1 after at least four 563 backcrosses to the B73 inbred line. Plants were grown in Supersoil Brand Potting Soil 564 (composted bark (60-85\%), Sawdust (60-85\%), Sphagnum Peat Moss (10-30\%)) in 565 standard greenhouse conditions. Seedlings were grown for 3 weeks in 30 by 45 by $5662.5 \mathrm{~cm}$ trays, and adult plants were grown in $12.5 \mathrm{~L}$ pots until tassel emergence. 567 Seedlings for the RNAseq analysis were the offspring of a cross between a normal 568 sibling and a mutant Oja sibling, grown in M3 soil mix with insecticide in large trays 569 equally spaced, in the Grodome facility at the University of Edinburgh with 570 supplementary lighting day/night cycles of 21C, 16hours / 15C, 8 hours.

\section{Root Analysis}

573 Seeds were rehydrated overnight in sterile water, then sterilized with $10 \%$ bleach for 20

574 minutes, before washing with sterile water. Seeds were then germinated on damp 575 chromotography paper in vertically fixed plastic wallets. After one week, the seedlings 576 were photographed, then rotated $90^{\circ}$. The seedlings were left to grow for 24 hours and 577 imaged every hour, for 12 hours. After 24 hours, the final root phenotype was imaged. 
578 Primary root length was analysed using FIJI (Schindelin et al., 2012) and crown root

579 number was counted. The seedlings were transplanted to soil and three weeks after 580 germination the shoot phenotype was recorded.

582 Histology Plastic Sections

$58310 \mu \mathrm{m}$ plastic sections were made with Technovitß 9100 from Electron Microscopy 584 Services (Cat. \#14655) according to manufacturer instructions. Sections were mounted 585 on glass slides and stained with toluidine blue. Imaging was performed on a Zeiss Axiovert inverted compound microscope with transmitted light.

\section{Scanning Electron Microscopy}

589 SEMs were performed with material fixed in FAA (3.7\% formaldehyde, 50\% ethanol, 5\% 590 acetic acid) overnight at $4^{\circ} \mathrm{C}$ and dehydrated in ethanol series to $100 \%$. Samples were 591 critical point dried, sputter coated with palladium, and imaged on a Hitachi S-4700 with $5922 \mathrm{kV}$ accelerating voltage.

Tissue for Immunolocalisation and In Situ Hybridisation

595 Plant tissue was collected from 3-week-old seedling shoot apices, dissected and fixed 596 overnight in 4\% paraformaldehyde/ $0.1 \%$ DMSO/ $0.1 \%$ Triton-X100. Fixed samples 597 were transferred through an ethanol and histoclear series before embedding in

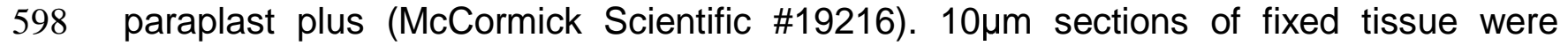
599 mounted on probe-on-probe-plus slides (FisherBrand \#22-230-900) and dried overnight 600 on a $37^{\circ} \mathrm{C}$ hotplate before use in either immunolocalisation or in situ hybridization.

602 Immunolocalisation

603 Primary antibodies were guinea-pig anti-SoPIN1 as used in (Richardson et al., 2016) 604 and rabbit anti-KNOTTED1 (KN1) as used in (Bolduc et al., 2012b). Secondary 605 antibodies were commercial anti-guinea pig conjugated to Alexa-fluor594 (Jackson 606 ImmunoResearch, \#706-585-148) and anti-rabbit alkaline phosphatase conjugate 607 (Jackson ImmunoResearch, \#111-055-045). All antibodies were used at a 1:200 dilution 608 in $1 \%$ bovine serum albumin (BSA) in PBS. 
Immunolocalisation used a protocol based on (Conti and Bradley, 2007), and was as follows. Slides were de-paraffinised using histoclear, then rehydrated through an

611 ethanol series to water. Samples were then boiled in 10mM sodium citrate, pH6, for 10 612 minutes to retrieve the antigens. Blocking was carried out in 1\% BSA/PBS/0.3\% Triton613 X100 for 3hours. Slides were incubated overnight in primary antibody, before washing in 614 PBS/0.3\% Triton-X100. They were then incubated with secondary antibody at room615 temperature for 2 hours.

616 Tissues incubated with anti-KN1 were incubated in a 1:50 dilution of NBT/BCIP 617 (Roche, \#11681451001) in 0.05M MgCl$/ 2 / \mathrm{TBS}$, pH9.5 until a dark precipitate was 618 observed. These slides were then imaged on a Leica dissecting microscope in water.

619 Tissues incubated with anti-SoPIN1 were stained for 20 minutes in $0.1 \%$ 620 calcofluor (fluorescent brightener 28, Sigma-Aldrich \#F3543), washed and mounted in 621 water and imaged on a Leica SP8 confocal microscope. The subcellular localization of 622 SoPIN1 was assessed in relation to the calcofluor cell wall stain using FIJI (Schindelin 623 et al., 2012).

\section{In situ Hybridisation}

626 Antisense probes targeted to DROOPLING LEAF1 (GRMZM2G168893) mRNA were 627 designed as used in (Strable et al., 2017). In situ hybridization was carried out as in 628 (Coen et al., 1990) outlined here in brief. Tissue was deparaffinised using histoclear, 629 then rehydrated through an ethanol series. Samples were digested using $0.125 \mathrm{mg} / \mathrm{mL}$ 630 pronase (Sigma-Aldrich \#P6611) in 50mMTris/5mM EDTA, pH7.5, for 15minutes at 631 room temperature. Digestion was stopped by the addition of $0.2 \%$ glycine before 632 washing and re-fixing in $4 \%$ PFA/PBS for 10 minutes. Slides were then treated in $0.1 \mathrm{M}$ 633 Triethanolamine-HCl / 0.5\% Acetic Anhydride (Sigma-Aldrich \#320102) for 10 minutes 634 before dehydrating through an ethanol series. Tissue was incubated overnight with the 635 probes at a 1:50 dilution in hybridisation buffer $(0.375 \mathrm{M} \mathrm{NaCl}, 12.5 \mathrm{mM}$ Tris- $\mathrm{HCl} \mathrm{pH}$, 636 12.5mM Sodium Phosphate pH6.8, 6.25mM EDTA, 50\% deionized formamide, 12.5\% 637 dextran sulfate, $1.25 x$ Denhardt solution, $0.0125 \mathrm{mg} / \mathrm{mL}$ tRNA) at $50^{\circ} \mathrm{C}$. Slides were 638 washed in $0.2 \% \mathrm{SSC}$ at $50^{\circ} \mathrm{C}$, then treated with RNAseA (in $0.5 \mathrm{M} \mathrm{NaCl} / 10 \mathrm{mM}$ Tris/ $6391 \mathrm{mM}$ EDTA, pH7.5) at $37^{\circ} \mathrm{C}$ for $15 \mathrm{mins}$, before repeating the SSC washes. Slides were 
640 blocked in Roche Blocking Reagent (\#11-096-176-001) for 30 minutes, washed in1\% 641 BSA/0.3 \% Triton-X100/TBS, and incubated at room-temperature with a 1:500 dilution 642 of anti-Dioxigenin-AP Fab fragments (Roche, \#11093274910) in 1\% BSA/0.3 \% Triton643 X100/TBS for 1hour. Slides were then washed with 1\% BSA/0.3 \% Triton-X100/TBS, 644 then in $0.05 \mathrm{M} \mathrm{MgCl}_{2} / \mathrm{TBS}, \mathrm{pH} 9.5$. To visualize the probe localisation, the tissue was 645 incubated in 1:50 dilution of NBT/BCIP in $0.05 \mathrm{M} \mathrm{MgCl} / \mathrm{TBS}, \mathrm{pH} 9.5$, until dark 646 precipitate formed. The staining reaction was stopped by transferring to water and the 647 slides were imaged on a Leica MZ16F microscope with a Qimaging Micropublisher 648 camera under water and brightfield conditions.

\section{Mapping with SSRs}

651 The Oja mutation was mapped to chromosome 3 using short sequence repeat (SSR) 652 PCR mapping. Known SSRs were identified using Maize gdb and are listed in Figure 3. 653 Custom SSRs were identified by using the Gramene SSR prediction tool (Temnykh et 654 al., 2001), and specific primers were designed to amplify them.

\section{Whole Genome Sequencing}

$65710 \mathrm{~mm}^{2}$ area leaf discs were collected from 30 wild type and 30 mutant siblings in an 658 advanced B73 introgressed population. Genomic DNA was extracted using a UREA 659 based extraction method (Chen and Dellaporta, 1994) which is as follows. Tissue was 660 ground in UREA extraction buffer (4M urea, 4M NaCl, 1M Tris pH8, 0.5M EDTA, 34mM 661 n-lauroyl sarcosine). Then an equal volume of phenol:chloroform:isoamyl alcohol 662 (25:24:1), was added and vortexed to mix. After centrifugation, the supernatant was 663 decanted and mixed with an equal volume of chloroform. After centrifugation, the 664 supernatant was decanted and mixed with $1 / 10^{\text {th }}$ volume of $4.4 \mathrm{M} \mathrm{NH}_{4} \mathrm{OAc} \mathrm{pH} 5.2$ and 6650.7 volume of isopropanol. Strands of DNA were then collected and washed with $70 \%$ 666 ethanol in a separate tube. The dried pellet was resuspended in 100ul of TE.

667 DNA was quantified using Qubit dsDNA HS Assay Kit (Invitrogen, Q32854), and 668 sent to Novogene for library preparation and sequencing (350bp insert, 150PE). 669 Sequencing was at 10 times coverage of the maize genome. Sequence analysis was 670 carried out using the CYVERSE Atmosphere computing environment. The sequence 
671 data was quality checked and the adapters trimmed using fastp, then aligned to the 672 B73_RefGen_v3 maize genome using bowtie2, variants were called using samtools 673 mileup. The variant list was filtered using $R$, by removing variants recorded in the 674 Panzea HapMapv3 (https://hapmap.ncbi.nlm.nih.gov/) and those present in the wild 675 type pool as well as restricting to EMS type SNPs. Moderate and high effect SNPs 676 specific to the mutant were calculated using SNPEff2 with the B73 RefGenv3 5b+gene 677 models.

RNASeq

680 Seedlings were imaged using a Canon EOS250D camera, then dissected and imaged 681 using a Leica MZ16F stereoscope with a Canon EOS250D camera attached via an LM682 Scope DSLR adapter. Seedlings were dissected at 21 and 28 days down to the 683 meristem and the P1/ P2 primordium, and individually flash frozen in liquid nitrogen. 684 RNA was extracted from each individual using TRIzol (Invitrogen), precipitated (0.5M $685 \mathrm{NaCl}+2$-propyl alcohol), and washed with 70\% ethanol, then resuspended in nuclease686 free water. RNA was quantified using a QuBit BR-RNA kit. RNA of sufficient quality was 687 sent to Novogene for 150bp paired-end sequencing, and 36 libraries passed quality 688 checks.

The CYVERSE Atmosphere virtual computing environment was used to carry out 690 sequence quality checks, genome alignments and count extraction. (4CPUs, 32G RAM, 691 with a ubuntu operating system). Sequenced libraries were checked for quality and 692 adapters were trimmed using fastp (Chen et al., 2018), then aligned to the B73 693 RefGenv3 genome using HiSAT2 (Kim et al., 2019). Counts were called using 694 FeatureCounts (Liao et al., 2014). All downstream analysis was carried out locally 695 (3.6GHz 8-Core Intel Core i9 processor, 32GB RAM) using R packages combined with 696 Jupyter notebooks. Differential gene expression analysis was carried out using DESeq2 697 (Love et al., 2014) using all genes defined as expressed (in more than 3 samples, with 698 more than 5 counts). Significantly expressed genes were defined as those with a padj 699 value of $<0.05$, and filtered to a log2 foldchange value of $<-0.59$ or $>0.59$. GO term 700 analysis used GOSeq (Young et al., 2010) with the maize-GAMER v3 annotations 
701 (Wimalanathan et al., 2018), and significantly enriched GO terms had a Benjamini and 702 Hochberg cutoff padj value of $<0.05$.

To carry out hierarchical clustering we stringently filtered the expressed gene list

704 to those genes with $>5$ counts in $>10$ samples, then filtered for those genes listed as 705 expressed in the meristem and leaf primordia from (Knauer et al., 2019). The top 1500 706 most variable of these genes were normalised and used to plot the 1 minus the absolute 707 value of the Pearson correlation matrix, and the resulting plot was clustered 708 hierarchically and cut using Kmeans $=4$. The samples in each cluster were then labelled 709 with cluster identity.

710 To generate self-organising maps, expressed genes (in more than 3 samples, 711 with more than 5 counts) were normalised, and the average variance stablised value 712 was calculated for each cluster. $4 \times 4$ node SOM grids were then generated using the 713 kohonen package (Wehrens and Kruisselbrink, 2018).

\section{Vein Analysis}

716 Cross-sections through the third leaf of 3 -week-old seedlings, $1.5 \mathrm{~cm}$ above the ligule 717 were taken and imaged under water on a Leica DM4000 microscope. Cross-section 718 images were analysed using FIJI (Schindelin et al., 2012). The width of the leaf was 719 recorded, then the number of lateral and intermediate veins were counted. Vein density 720 was calculated as the total number of veins (lateral + intermediate veins) over the width 721 of the leaf.

722 Vein distribution was assessed by counting the number of intermediate veins 723 between lateral veins. To align the wild type and midribless leaves, the lateral vein 724 closest to the midpoint of the leaf was designated at position 0, the "midvein". Each 725 lateral vein was then allocated a position number in reference to position 0. 
727 Acknowledgements: With thanks to Samuel Leiboff and China Lunde for extensive 728 help and advice, ARS microscopy staff, UC Berkeley greenhouse staff, and the 729 University of Edinburgh Plant Growth Facilities. Author Contributions: SH conceived 730 the original project, developed the mapping population and secured funding. AR and AS 731 designed and carried out the experiments. AR and SH wrote the manuscript. AR agrees

732 to serve as the author responsible for contact and ensures communication. Funding: 733 This work was funded by NSF BIO/BBSRC1547062, ARS 5335-21000-013-00D and 734 AR's University of Edinburgh Start-Up funds. This work made use of material supported 735 by the National Science Foundation under Award Numbers DBI-0735191, DBI-1265383, 736 and DBI-1743442 (www.cyverse.org) Materials and Data Availability: All microscopy 737 data is available via the University of Edinburgh DataShare 738 (https://datashare.ed.ac.uk/handle/10283/3987) in The Plant Shape Lab collection. All 739 next generation sequencing data is available via MaizeGDB or the University of 740 Edinburgh DataShare in The Plant Shape Lab collection. Jupyter notebooks for the 741 bioinformatic analyses are available via Github

742 (https://github.com/ThePlantShapeLab/Hojaloca1).

743 All materials are available on request from the corresponding author. All other data are 744 in the main paper or the supplement.

Figures 1-7

749 Supplementary Table 1: Custom SSR mapping primers

750 Supplementary Figures 1-3

751 Supplementary Datasets 1-5 
Aida, M., Ishida, T., and Tasaka, M. (1999). Shoot apical meristem and cotyledon COTYLEDON and SHOOT MERISTEMLESS genes. Development 126: 15631570.

Barazesh, S., Nowbakht, C., and McSteen, P. (2009). sparse inflorescence1, barren inflorescence1 and barren stalk1 promote cell elongation in maize inflorescence development. Genetics 182: 403-406.

Bayer, E.M., Smith, R.S., Mandel, T., Nakayama, N., Sauer, M., Prusinkiewicz, P., and Kuhlemeier, C. (2009). Integration of transport-based models for phyllotaxis and midvein formation. Genes Dev. 23: 373-384.

Baylis, T., Cierlik, I., Sundberg, E., and Mattsson, J. (2013). SHORT INTERNODES/STYLISH genes, regulators of auxin biosynthesis, are involved in leaf vein development in Arabidopsis thaliana. New Phytol. 197: 737-750.

Becraft, P.W. and Freeling, M. (1991). Sectors of liguleless-1 Tissue Interrupt an Inductive Signal during Maize Leaf Development. Plant Cell.

Von Behrens, I., Komatsu, M., Zhang, Y., Berendzen, K.W., Niu, X., Sakai, H., Taramino, G., and Hochholdinger, F. (2011). Rootless with undetectable meristem 1 encodes a monocot-specific AUX/IAA protein that controls embryonic seminal and post-embryonic lateral root initiation in maize. Plant J.

Benková, E., Michniewicz, M., Sauer, M., Teichmann, T., Seifertová, D., Jürgens, G., and Friml, J. (2003). Local, efflux-dependent auxin gradients as a common module for plant organ formation. Cell 115: 591-602.

Bhatia, N., Bozorg, B., Larsson, A., Ohno, C., Jönsson, H., and Heisler, M.G. (2016). Auxin acts through MONOPTEROS to regulate plant cell polarity and pattern phyllotaxis. Curr. Biol. 26: 3202-3208.

Bolduc, N., O’Connor, D., Moon, J., Lewis, M., and Hake, S. (2012a). How to pattern a leaf. In Cold Spring Harbor symposia on quantitative biology (Cold Spring Harbor Laboratory Press), pp. 47-51.

Bolduc, N., Yilmaz, A., Mejia-Guerra, M.K., Morohashi, K., O’Connor, D., Grotewold, E., and Hake, S. (2012b). Unraveling the KNOTTED1 regulatory 
network in maize meristems. Genes Dev. 26: 1685-1690.

Chen, J. and Dellaporta, S. (1994). Urea-based plant DNA miniprep. In The maize handbook (Springer), pp. 526-527.

792 Chen, S., Zhou, Y., Chen, Y., and Gu, J. (2018). Fastp: An ultra-fast all-in-one FASTQ preprocessor. Fastp An ultra-fast all-in-one FASTQ preprocessor.

Coen, E.S., Romero, J., Doyle, S., Elliott, R., Murphy, G., and Carpenter, R. (1990). floricaula: a homeotic gene required for flower development in Antirrhinum majus. Cell 63: 1311-1322.

Conklin, P.A., Johnston, R., Conlon, B.R., Shimizu, R., and Scanlon, M.J. (2021). Plant homeodomain proteins provide a mechanism for how leaves grow wide. Dev.

Conti, L. and Bradley, D. (2007). TERMINAL FLOWER1 is a mobile signal controlling Arabidopsis architecture. Plant Cell 19: 767-778.

Dharmasiri, N., Dharmasiri, S., and Estelle, M. (2005). The F-box protein TIR1 is an auxin receptor. Nature 435: 441-445.

Fukaki, H., Tameda, S., Masuda, H., and Tasaka, M. (2002). Lateral root formation is

Galinat, W.C. (1959). The phytomer in relation to floral homologies in the American Maydeae. Bot. Mus. Lealf. Harv. Univ. 19: 1-xvi.

Galli, M., Khakhar, A., Lu, Z., Chen, Z., Sen, S., Joshi, T., Nemhauser, J.L., Schmitz, R.J., and Gallavotti, A. (2018). The DNA binding landscape of the maize AUXIN RESPONSE FACTOR family. Nat. Commun. 9: 1-14.

Hamann, T., Benkova, E., Bäurle, I., Kientz, M., and Jürgens, G. (2002). The Arabidopsis BODENLOS gene encodes an auxin response protein inhibiting MONOPTEROS-mediated embryo patterning. Genes Dev. 16: 1610-1615. during ligule induction in maize. Genetics 144: 1871-1882. 
Havens, K.A., Guseman, J.M., Jang, S.S., Pierre-Jerome, E., Bolten, N., Klavins, E., and Nemhauser, J.L. (2012). A synthetic approach reveals extensive tunability of auxin signaling. Plant Physiol. 160: 135-142. M. (2006). Arabidopsis CUP-SHAPED COTYLEDON3 regulates postembryonic shoot meristem and organ boundary formation. Plant Cell 18: 2946-2957. homeobox genes in the shoot apical meristem predicts patterns of morphogenesis in the vegetative shoot. Development 120: 405-413.

Johnston, R., Leiboff, S., and Scanlon, M.J. (2015). Ontogeny of the sheathing leaf base in maize (Zea mays). New Phytol. 205: 306-315.

Johnston, R., Wang, M., Sun, Q., Sylvester, A.W., Hake, S., and Scanlon, M.J. (2014). Transcriptomic analyses indicate that maize ligule development

Kepinski, S. and Leyser, O. (2005). The Arabidopsis F-box protein TIR1 is an auxin receptor. Nature 435: 446-451.

Kim, D., Paggi, J.M., Park, C., Bennett, C., and Salzberg, S.L. (2019). Graph-based recapitulates gene expression patterns that occur during lateral organ initiation. genome alignment and genotyping with HISAT2 and HISAT-genotype. Nat. Biotechnol.

Knauer, S. et al. (2019). A high-resolution gene expression atlas links dedicated meristem genes to key architectural traits. Genome Res.

Kuhlemeier, C. and Timmermans, M.C.P. (2016). The Sussex signal: insights into leaf dorsiventrality. Development 143: 3230-3237.

Lavy, M. and Estelle, M. (2016). Mechanisms of auxin signaling. Development 143: 3226-3229.

Leyser, H.M.O., Pickett, F.B., Dharmasiri, S., and Estelle, M. (1996). Mutations in the AXR3 gene of Arabidopsis result in altered auxin response including ectopic expression from the SAUR-AC1 promoter. Plant J. 10: 403-413.

Liao, Y., Smyth, G.K., and Shi, W. (2014). FeatureCounts: An efficient general purpose program for assigning sequence reads to genomic features. 
Bioinformatics.

Love, M.I., Huber, W., and Anders, S. (2014). Moderated estimation of fold change and dispersion for RNA-seq data with DESeq2. Genome Biol.

Matthes, M.S., Best, N.B., Robil, J.M., Malcomber, S., Gallavotti, A., and McSteen, P. (2019). Auxin EvoDevo: conservation and diversification of genes regulating auxin biosynthesis, transport, and signaling. Mol. Plant 12: 298-320.

McSteen, P. and Hake, S. (2001). Barren inflorescence2 regulates axillary meristem development in the maize inflorescence. Development.

Moon, J., Candela, H., and Hake, S. (2013). The Liguleless narrow mutation affects proximal-distal signaling and leaf growth. Development 140: 405-412.

Nagpal, P., Walker, L.M., Young, J.C., Sonawala, A., Timpte, C., Estelle, M., and Reed, J.W. (2000). AXR2 encodes a member of the Aux/IAA protein family. Plant Physiol. 123: 563-574.

Nardmann, J., Ji, J., Werr, W., and Scanlon, M.J. (2004). The maize duplicate genes narrow sheath1 and narrow sheath2 encode a conserved homeobox gene function in a lateral domain of shoot apical meristems. Development 131: 2827-2839.

Nogueira, F.T.S., Madi, S., Chitwood, D.H., Juarez, M.T., and Timmermans, M.C.P. (2007). Two small regulatory RNAs establish opposing fates of a developmental axis. Genes Dev. 21: 750-755.

O’Connor, D.L., Elton, S., Ticchiarelli, F., Hsia, M.M., Vogel, J.P., and Leyser, T. (2017). Cross-species functional diversity within the PIN auxin efflux protein family. Elife.

O’Connor, D.L., Runions, A., Sluis, A., Bragg, J., Vogel, J.P., Prusinkiewicz, P., and Hake, S. (2014). A division in PIN-mediated auxin patterning during organ initiation in grasses. PLoS Comput Biol 10: e1003447.

Ouellet, F., Overvoorde, P.J., and Theologis, A. (2001). IAA17/AXR3: biochemical insight into an auxin mutant phenotype. Plant Cell 13: 829-841.

Overvoorde, P.J., Okushima, Y., Alonso, J.M., Chan, A., Chang, C., Ecker, J.R., Hughes, B., Liu, A., Onodera, C., and Quach, H. (2005). Functional genomic analysis of the AUXIN/INDOLE-3-ACETIC ACID gene family members in Arabidopsis thaliana. Plant Cell 17: 3282-3300. 
Parry, G., Calderon-Villalobos, L.I., Prigge, M., Peret, B., Dharmasiri, S., Itoh, H., Lechner, E., Gray, W.M., Bennett, M., and Estelle, M. (2009). Complex regulation of the TIR1/AFB family of auxin receptors. Proc. Natl. Acad. Sci. 106: 22540 LP 22545.

Pérez-Pérez, J.M., Candela, H., Robles, P., López-Torrejón, G., Del Pozo, J.C., and Micol, J.L. (2010). A role for AUXIN RESISTANT3 in the coordination of leaf growth. Plant cell Physiol. 51: 1661-1673.

Powers, S.K. and Strader, L.C. (2020). Regulation of auxin transcriptional responses. Dev. Dyn. 249: 483-495.

Ramos, J.A., Zenser, N., Leyser, O., and Callis, J. (2001). Rapid degradation of auxin/indoleacetic acid proteins requires conserved amino acids of domain II and is proteasome dependent. Plant Cell 13: 2349-2360.

Reed, J.W. (2001). Roles and activities of Aux/IAA proteins in Arabidopsis. Trends Plant Sci. 6: 420-425.

Reed, J.W., Elumalai, R.P., and Chory, J. (1998). Suppressors of an Arabidopsis thaliana phyB mutation identify genes that control light signaling and hypocotyl elongation. Genetics 148: 1295-1310.

Reinhardt, D., Pesce, E.-R., Stieger, P., Mandel, T., Baltensperger, K., Bennett, M., Traas, J., Friml, J., and Kuhlemeier, C. (2003). Regulation of phyllotaxis by polar auxin transport. Nature 426: 255-260.

Richardson, A.E. and Hake, S. (2019). Drawing a line: Grasses and boundaries. Plants 8.

Richardson, A.E., Rebocho, A.B.A.B., and Coen, E.S. (2016). Ectopic KNOX expression affects plant development by altering tissue cell polarity and identity. Plant Cell 28: tpc-00284.

Rogg, L.E., Lasswell, J., and Bartel, B. (2001). A gain-of-function mutation in IAA28 suppresses lateral root development. Plant Cell 13: 465-480.

Rouse, D., Mackay, P., Stirnberg, P., Estelle, M., and Leyser, O. (1998). Changes in auxin response from mutations in an AUX/IAA gene. Science (80-. ). 279: 13711373.

Salehin, M., Bagchi, R., and Estelle, M. (2015). SCFTIR1/AFB-based auxin 
perception: mechanism and role in plant growth and development. Plant Cell 27: 919.

Scanlon, M.J. (2003). The polar auxin transport inhibitor N-1-naphthylphthalamic acid disrupts leaf initiation, KNOX protein regulation, and formation of leaf margins in maize. Plant Physiol. 133: 597-605.

Scanlon, M.J. and Freeling, M. (1997). Clonal sectors reveal that a specific meristematic domain is not utilized in the maize mutantnarrow sheath. Dev. Biol. 182: $52-66$.

Sharman, B.C. (1942). Developmental anatomy of the shoot of Zea mays L. Ann. Bot. 6: 245-282.

Singh, S., Yadav, S., Singh, A., Mahima, M., Singh, A., Gautam, V., and Sarkar, A.K. (2020). Auxin signaling modulates LATERAL ROOT PRIMORDIUM1 (LRP1) expression during lateral root development in Arabidopsis. Plant J. 101: 87-100.

Strable, J., Wallace, J.G., Unger-Wallace, E., Briggs, S., Bradbury, P.J., Buckler, E.S., and Vollbrecht, E. (2017). Maize YABBY genes drooping leaf1 and drooping leaf2 regulate plant architecture. Plant Cell 29: 1622-1641.

Tan, X., Calderon-Villalobos, L.I.A., Sharon, M., Zheng, C., Robinson, C. V, Estelle, Smith, L.G., Greene, B., Veit, B., and Hake, S. (1992). A dominant mutation in the maize homeobox gene, Knotted-1, causes its ectopic expression in leaf cells with altered fates. Development 116: 21-30.

M., and Zheng, N. (2007). Mechanism of auxin perception by the TIR1 ubiquitin ligase. Nature 446: 640-645.

Temnykh, S., DeClerck, G., Lukashova, A., Lipovich, L., Cartinhour, S., and McCouch, S. (2001). Computational and experimental analysis of microsatellites in rice (Oryza sativa L.): frequency, length variation, transposon associations, and genetic marker potential. Genome Res. 11: 1441-1452.

Tian, Q., Nagpal, P., and Reed, J.W. (2003). Regulation of Arabidopsis SHY2/IAA3 protein turnover. Plant J. 36: 643-651. 
944 Tian, Q. and Reed, J.W. (1999). Control of auxin-regulated root development by the Arabidopsis thaliana SHY2/AA3 gene. Development.

946 Tsuda, K., Abraham-Juarez, M.-J., Maeno, A., Dong, Z., Aromdee, D., Meeley, R., Shiroishi, T., Nonomura, K., and Hake, S. (2017). KNOTTED1 cofactors, BLH12 and $\mathrm{BLH14}$, regulate internode patterning and vein anastomosis in maize. Plant Cell 29: 1105-1118.

Wang, H., Jones, B., Li, Z., Frasse, P., Delalande, C., Regad, F., Chaabouni, S., Latché, A., Pech, J.C., and Bouzayen, M. (2005). The tomato Aux/IAA transcription factor IAA9 is involved in fruit development and leaf morphogenesis. Plant Cell.

Wehrens, R. and Kruisselbrink, J. (2018). Flexible self-organizing maps in kohonen 3.0. J. Stat. Softw.

Wimalanathan, K., Friedberg, I., Andorf, C.M., and Lawrence-Dill, C.J. (2018). Maize GO Annotation-Methods, Evaluation, and Review (maize-GAMER). Plant Direct.

Woll, K., Borsuk, L.A., Stransky, H., Nettleton, D., Schnable, P.S., and Hochholdinger, F. (2005). Isolation, characterization, and pericycle-specific transcriptome analyses of the novel maize lateral and seminal root initiation mutant rum1. Plant Physiol.

Worley, C.K., Zenser, N., Ramos, J., Rouse, D., Leyser, O., Theologis, A., and Callis, J. (2000). Degradation of Aux/IAA proteins is essential for normal auxin signalling. Plant J.

Yang, X., Lee, S., So, J.H., Dharmasiri, S., Dharmasiri, N., Ge, L., Jensen, C., Hangarter, R., Hobbie, L., and Estelle, M. (2004). The IAA1 protein is encoded by AXR5 and is a substrate of SCF TIR1. Plant J.

Young, M.D., Wakefield, M.J., Smyth, G.K., and Oshlack, A. (2010). Gene ontology analysis for RNA-seq: accounting for selection bias. Genome Biol.

Zenser, N., Ellsmore, A., Leasure, C., and Callis, J. (2001). Auxin modulates the degradation rate of Aux/IAA proteins. Proc. Natl. Acad. Sci. U. S. A.

Zhang, Y., Marcon, C., Tai, H., Von Behrens, I., Ludwig, Y., Hey, S., Berendzen, K.W., and Hochholdinger, F. (2016). Conserved and unique features of the 

MERISTEM 1 and RUM1-like 1. J. Exp. Bot.

977 Zhang, Z., Runions, A., Mentink, R.A., Kierzkowski, D., Karady, M., Hashemi, B., 978 Huijser, P., Strauss, S., Gan, X., and Ljung, K. (2020). A WOX/Auxin

979 Biosynthesis Module Controls Growth to Shape Leaf Form. Curr. Biol. 

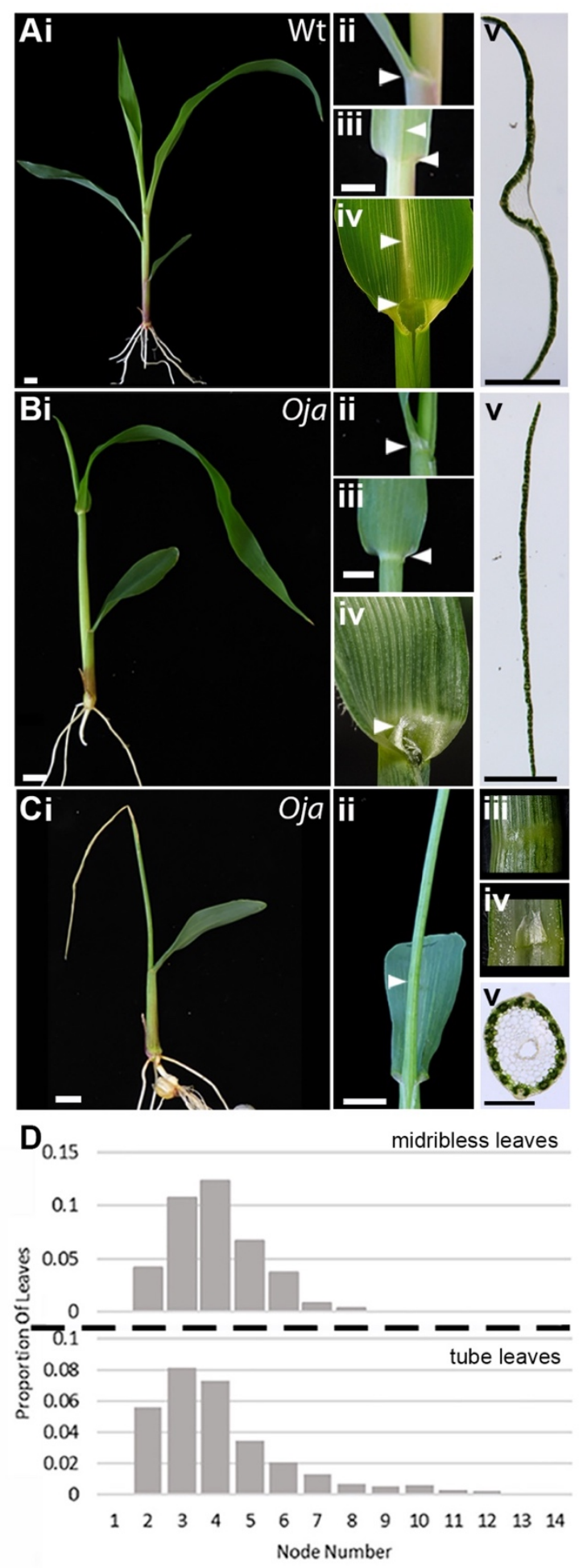

983 Figure 1. Hoja loca1 mutants have leaf defects.

984 The phenotype of wild type (Wt) (A), mild (B) and severe (C) Oja mutants including (i) 985 whole-seedling, and (ii-iv) magnified images of the ligule/ auricle regions, showing the 986 side (ii) and abaxial (iii) and the adaxial (iv) views. (C iv) is a cross-section through the 987 tube leaf showing the intact ligule (arrowhead in Cii). (v) Cross-sections through 
988 seedling leaves $1 \mathrm{~cm}$ above the ligule. Scale bars are $1 \mathrm{~cm}$, arrowheads indicate the 989 ligule or midrib regions. (D) The distribution of midribless and tube leaves in Oja plants $990 \quad(n=150)$, node 1 is at the base of the plant.

991 
A

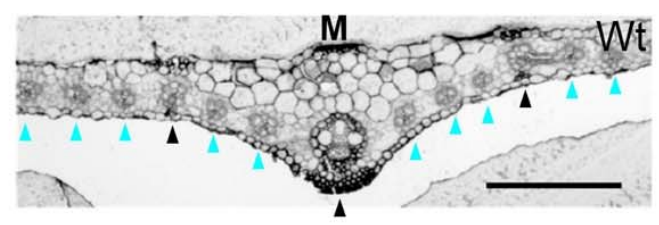

B
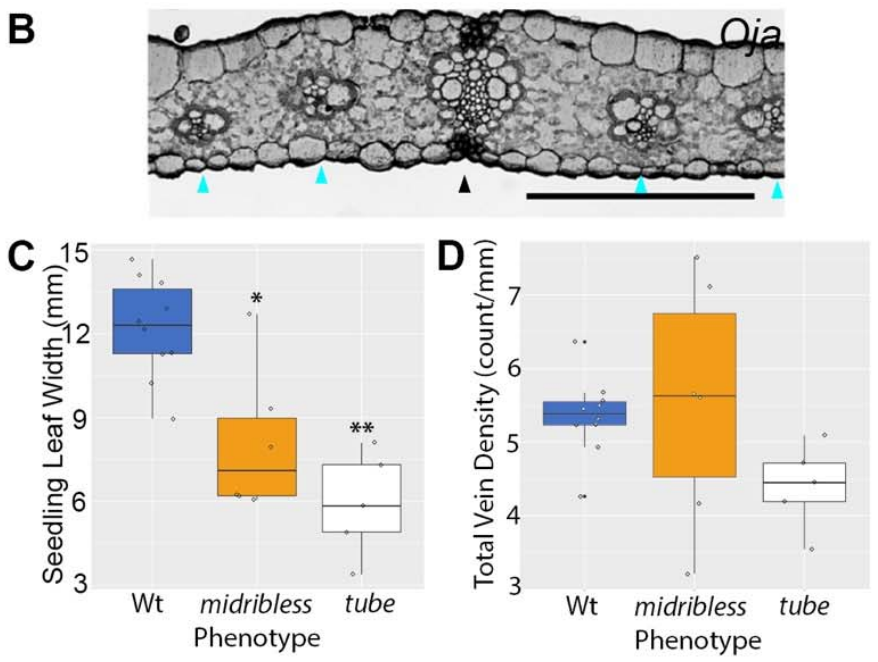

$E_{\mathbf{i}}$

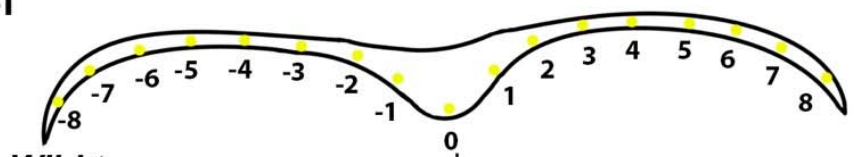

Wild type

Midvein

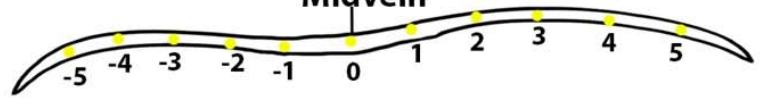

Oja midribless

ii

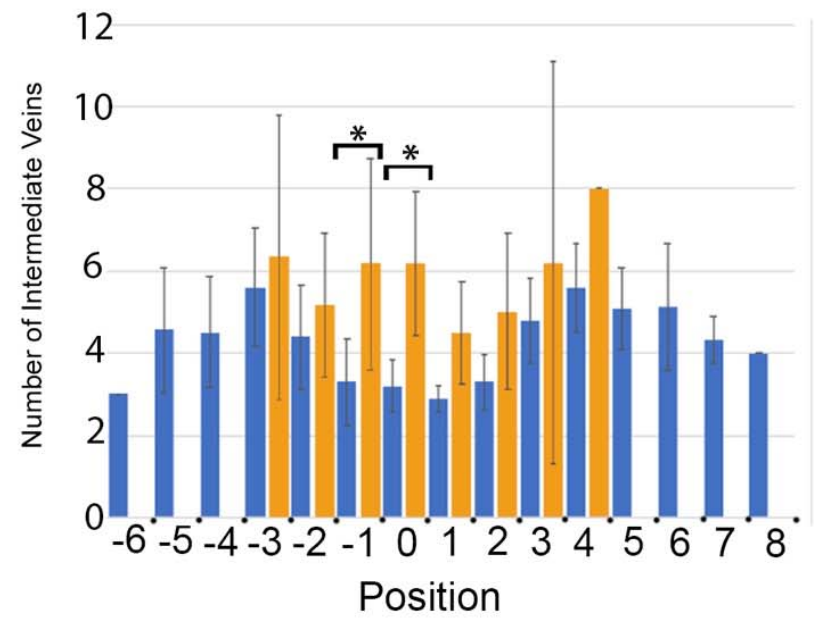

\section{Position}

Figure 2. Hoja loca1 mutants have a defect in vein distribution. 
$\left.998 \mathrm{df}=2,{ }^{*} \mathrm{p}=0.0032\right)$ and between wild type and tube leaves $\left({ }^{* *}, p=0.000083\right)$. (D) Total 999 leaf vein density does not vary significantly. (E) Leaf vein distribution is perturbed in 1000 midribless mutants. (Ei) Cartoons depicting the alignment of midribless and wild-type 1001 leaves. Wild type and mutant leaves were aligned by allocating the position of "0" to the 1002 wild type midvein, and the lateral vein closest to the midpoint of the leaf in the 1003 midribless mutant. Each successive lateral vein was then allocated a position relative to 10040 (Ei) and the number of intermediate veins between each position was recorded. (Eii) 1005 The mean number of intermediate veins near the midvein were significantly different in 1006 the midribless mutant (orange) when compared to wild type (blue) ( $n=10$ for each 1007 1008 phenotype, ANOVA and Tukeys HSD, ${ }^{*} p=0.0051$ and $p=0.00089$ ). 

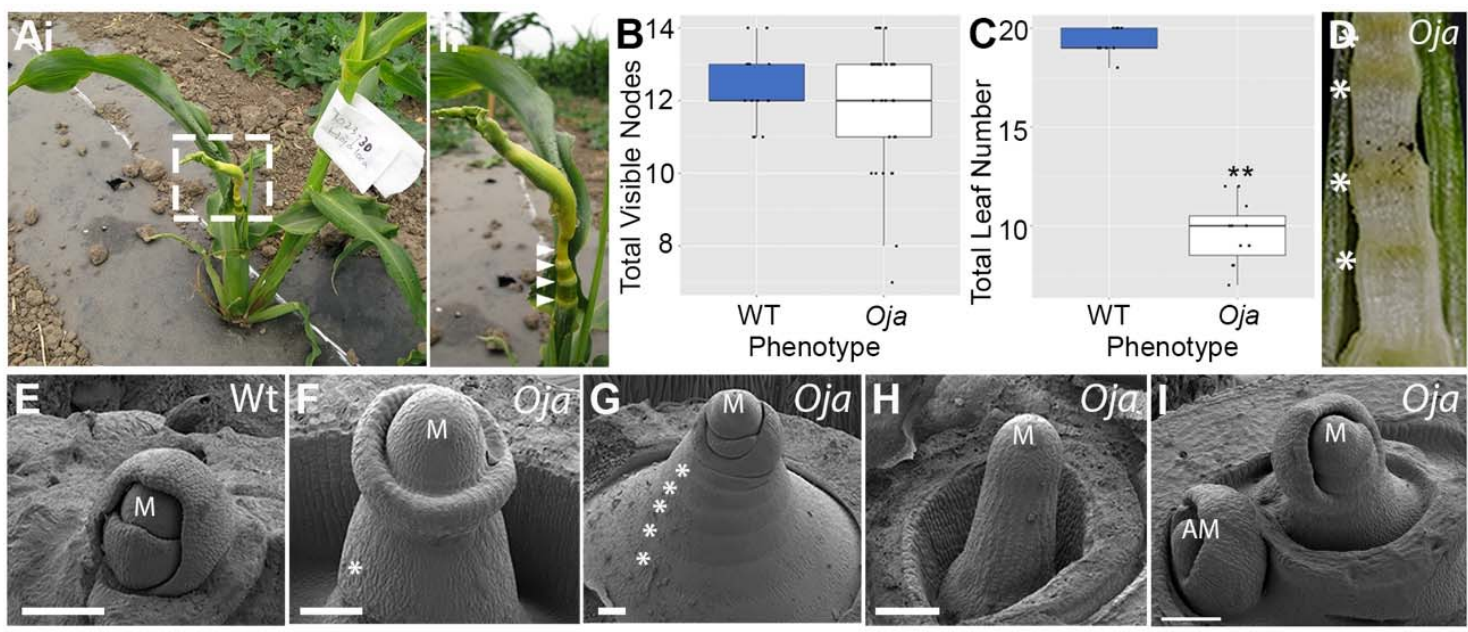

Figure 3. Hoja loca1 mutants initiate new phytomers but fail to initiate leaves. nodes $(B, p=0.2537$, Welch's 2-Sample T-Test, $d f=39.8, n$ Oja $=29, \mathrm{Wt}=14)$, they have fewer leaves $\left(C,{ }^{* *} p=4.17 \mathrm{e}-11\right.$, Welch's 2-Sample T-Test, $\mathrm{df}=14.194, \mathrm{n}=10$ of each phenotype) than wild type. In particular, the nodes below the tassel are often bare (Aii, arrowheads). Bare nodes can also be identified in cross-sections through developing shoots (D). The lack of leaf initiation is clear at the earliest stages of development. Scanning electron micrographs (SEMs) of wild type (E) and mutant (F-I) vegetative meristems with attached leaf primordia show severe defects in Oja. Some leaf primordia fail to arch over the meristem, instead forming a ring-shaped primordium (F). Some have clear ridges where internodes have formed below the meristem $(G)$, which would normally be concealed by leaves in wild-type plants. Other mutants form pin-shaped meristems $(\mathrm{H})$. Often the tiller buds in Oja are normal (I) allowing the plant to produce progeny. The internodes are indicated by the *, meristems by ' $M$ ', axillary meristems by 


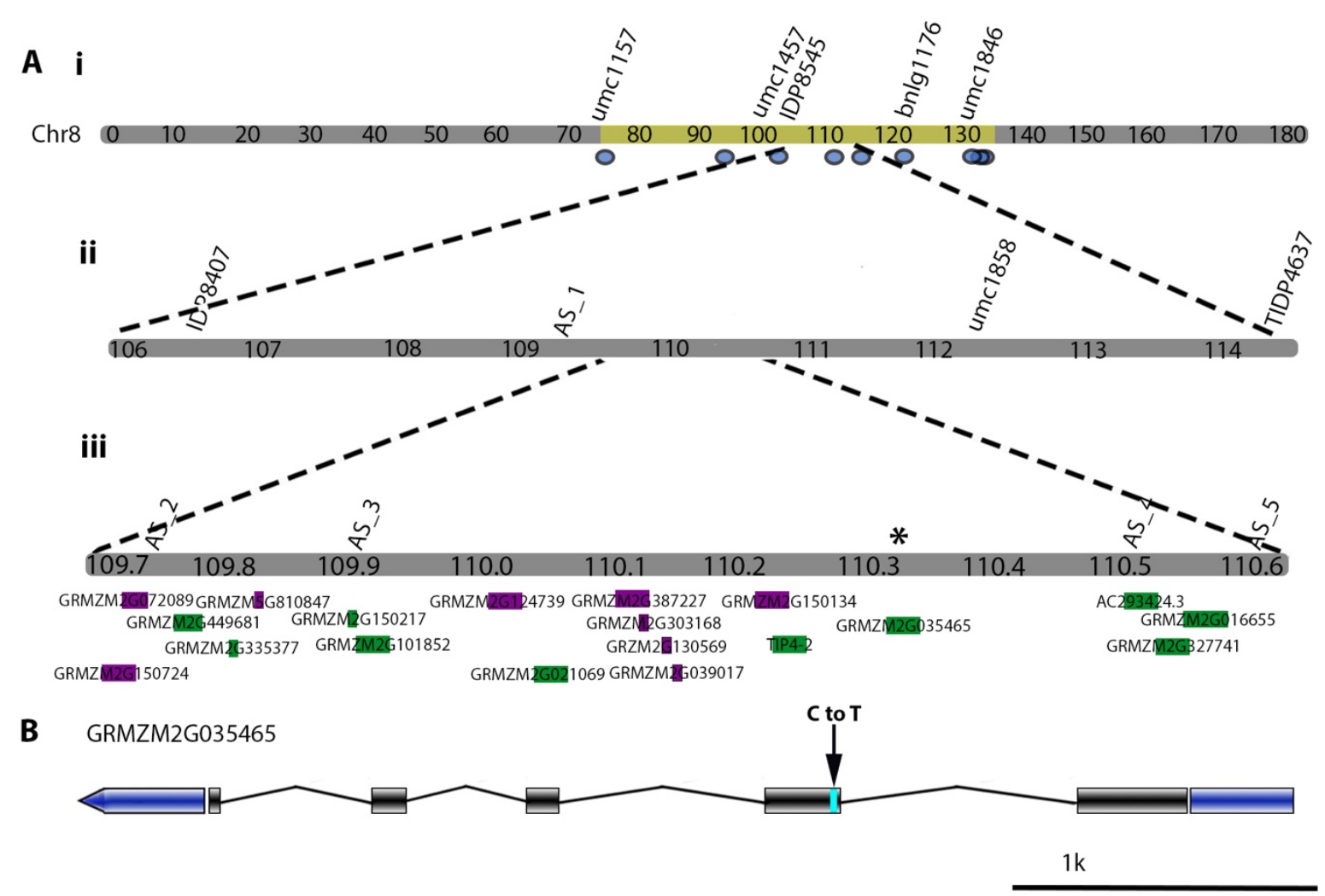

C ZmIAA28_GRMZM2G035465 EYDEKKVAATPQPAAKAQVVGWPPVCNYRKNTM 120 ZmIAA28_GRMZM2G035465_Oja EYDEKKVAATPQPAAKAQVVENPPVCNYRKNTM 120 HORVU3Hr1G031460 OsIAA17_Os05g0230700 AtPAP2_A At4G29080 SIIAA8_Solyc12g007230 DEKDKKVAAPPQPAAKAQVVGWPPIRSYRKNTM 98 EEDKKVAAAPQAPAAKAQVVGWPPIRSYRKNTM 131 ----EKKSSATAPASKAQVVGWPPIRSFRKNSM 158 ALICSTNSHGVAPAAKAQVVGWPPIRSFRKNMV 158

1026 Figure 4. Hoja loca1 maps to ZmIAA28 on Chr8.

1027 Using short sequence repeat (SSR) PCR markers, Hoja was mapped to Chr8 between $1028109.7-110.6 \mathrm{Mb}$ (A). Custom SSR markers are labeled as 'AS\#'. Of the 19 genes in the 1029 fine mapping interval (Aiii), 10 are expressed in shoot meristems and leaf primordia 1030 (green), 9 are not (magenta) based on published RNAseq datasets. Whole genome sequencing (WGS) data identified 9 high to moderate effect SNPs in gene bodies in the larger mapping region (Al, blue ovals, Chr8:75-135Mb). Of these, only one is an EMStype SNP that falls within the fine mapping interval (Aiii, ${ }^{*}$ ). (B)The SNP causes a C to T change in the second exon of GRMZM2G035465, ZmIAA28 which results in a glycine (G) to glutamic acid (E) amino acid change in the highly conserved core degron motif (C, cyan box). 
A
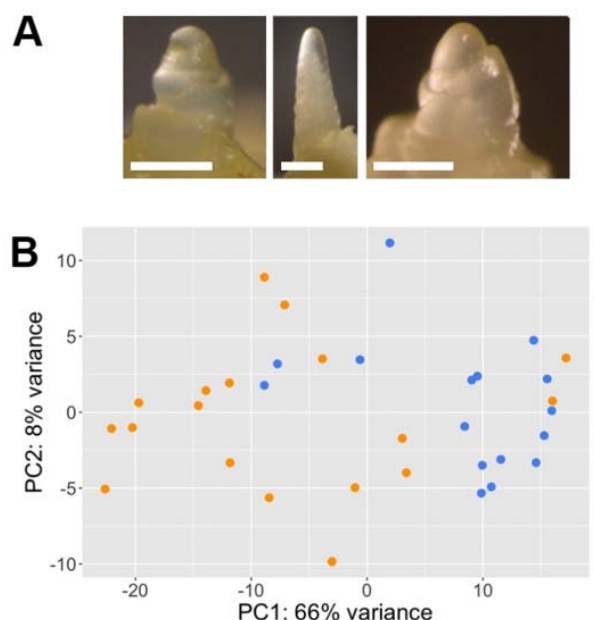

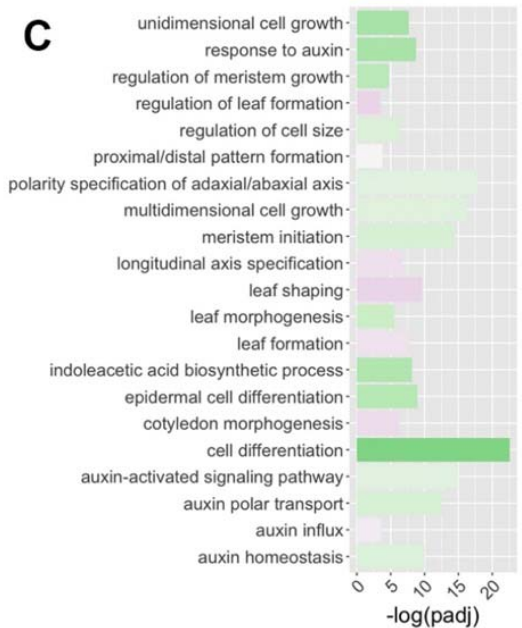

1039 Figure 5. Hoja loca1 mutants show changes in auxin, leaf development, and 1040 meristem regulation genes.

(A) Examples of the dissected shoot apical meristems harvested for the RNAseq experiment. Scalebar:100um. (B) Principal component analysis plot (PCA) of all RNAseq libraries, coloured by seeding phenotype at time of harvest; mutant (orange) or wild-type (blue) $(n=100)$. (C) Significantly enriched GO terms (-log(padj) $>1)$ associated with auxin, meristem regulation and leaf development for all genes that are significantly differentially expressed between mutant and wild type scored samples (padj $<0.05$, log2foldchange $>0.59$ or $<-0.59)$. Z-score indicates whether the genes in the GO term are on average upregulated (green) or downregulated (purple). 

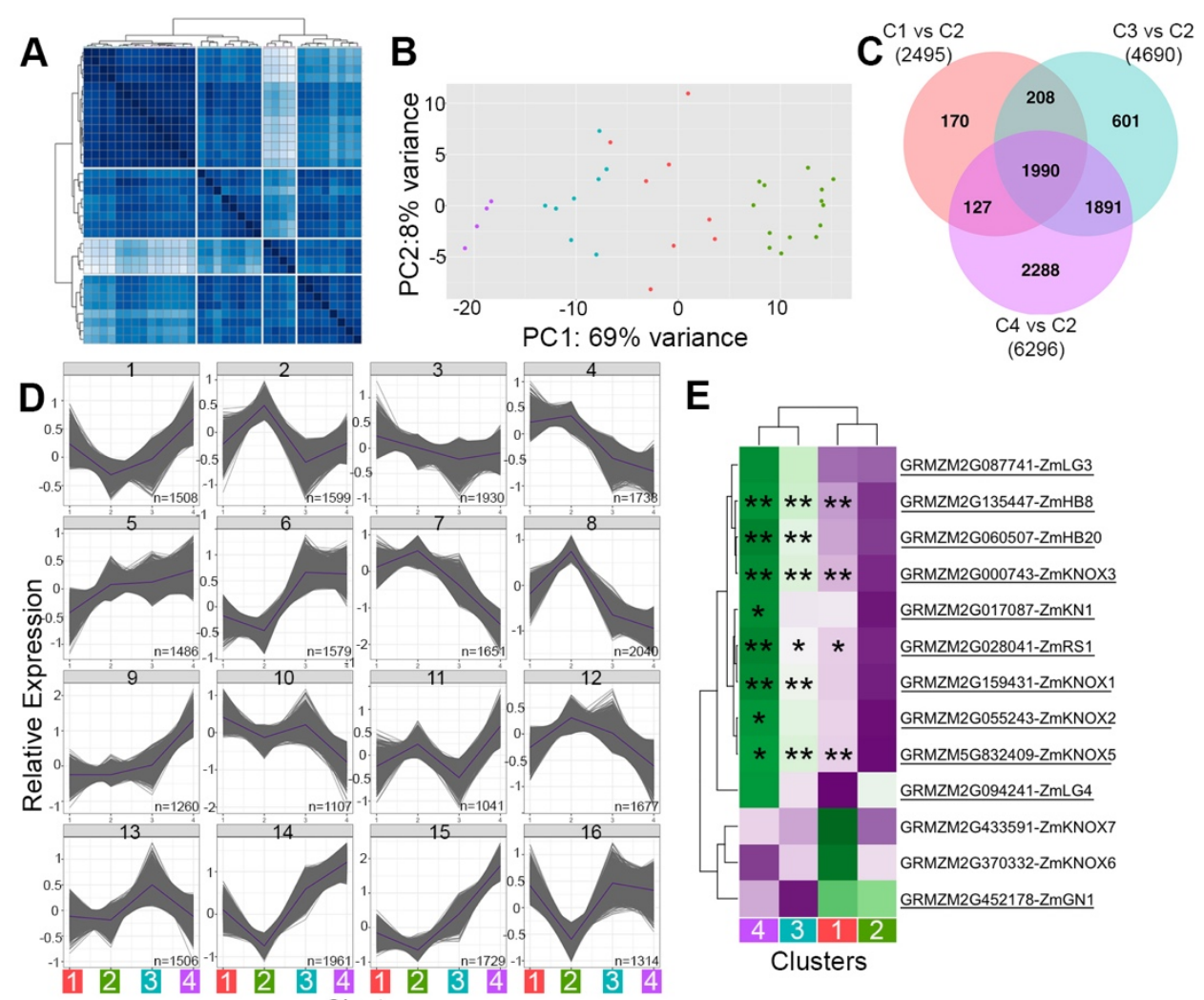

E

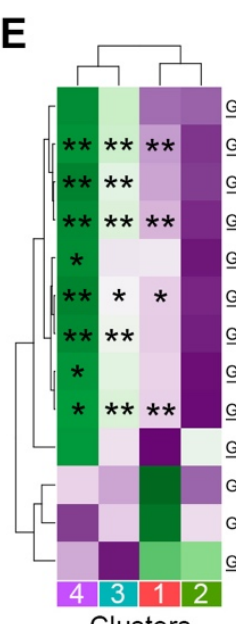

GRMZM26087741-ZmLG3

GRMZM2G135447-ZmHB8 GRMZM2G060507-ZmHB20 GRMZM2G000743-ZmKNOX3 GRMZM2 6017087-ZmKN1 GRMZM2 6028041-ZmRS1 GRMZM2G159431-ZmKNOX1 GRMZM2G055243-ZmKNOX2 GRMZM5G832409-ZmKNOX5 GRMZM2 G094241-ZmLG4 GRMZM2G433591-ZmKNOX7 GRMZM2G370332-ZmKNOX6 GRMZM2G452178-ZmGN1

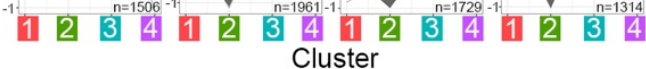
Clusters
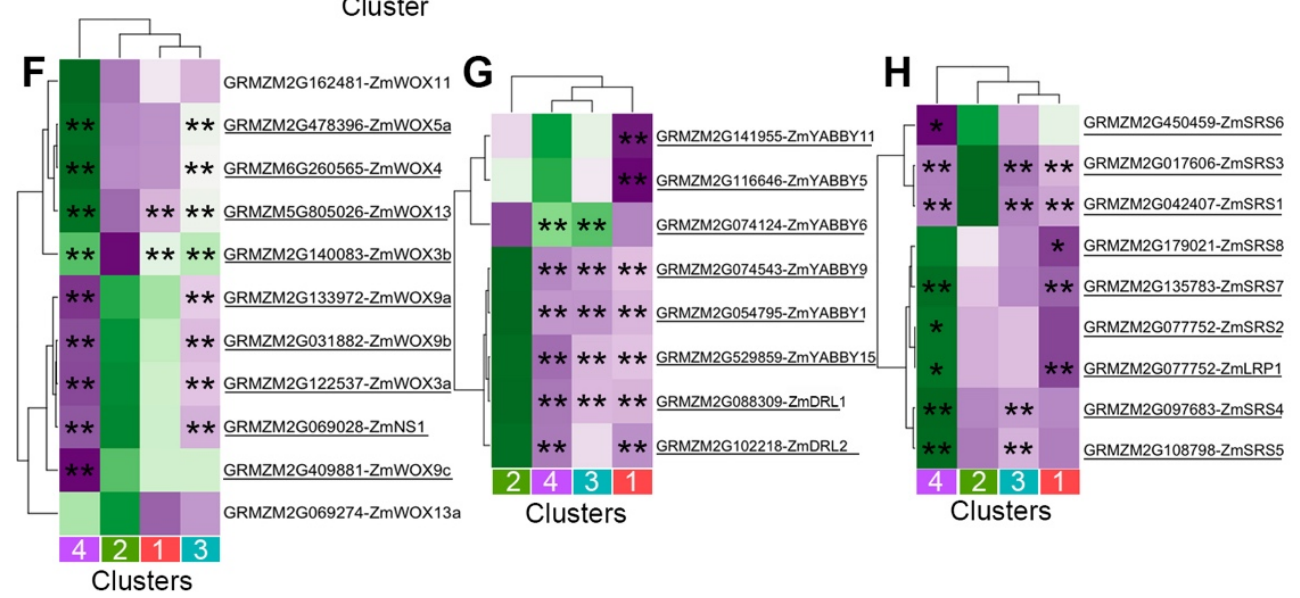
Figure 6. Transcriptional profiles of individual vegetative meristems enable identification of four transcriptional profiles phenotypes.

(A) Hierarchical clustering of 1 minus the Pearsons correlation of sample distances

1054 (dark blue most similar) based on the top 1500 most variable leaf and meristem associated expressed genes. $K=4$. (B) Principal component analysis plot (PCA) coloured by the 4 clusters identified in the hierarchical clustering $(n=100)$, cluster 1 (red), cluster 2 (green), cluster 3 (blue), cluster 4 (purple). (C) Venn diagram showing the overlap in significantly differentially expressed (sigDE) genes in comparisons with cluster 2 (C2) which have a log2foldchange of $>0.59$ or $<-0.59$. C\#vsC\# indicates cluster comparison. (\#) indicate the total number of sigDE genes, numbers indicate the number 
1061 of genes in each category. (D) $4 \times 4$ self-organizing maps built from all expressed genes 1062 (defined as more than $5 \mathrm{rpm}$ in more than 3 samples) in each cluster. Nodes 1-16 are 1063 shown. $\mathrm{N}$ indicates number of genes in the node. $(\mathrm{E}-\mathrm{H})$ Heatmaps of average FPKM 1064 values of expressed KNOX $(\mathrm{E})$, WOX $(\mathrm{F}), \operatorname{YABBY}(\mathrm{G})$ and $S R S(\mathrm{H})$ genes in clusters 11065 4. Colouring is relative average FPKM per row, green is positive, purple is negative. 1066 Genes that are significantly differentially expressed in one or more pairwise 1067 comparisons with cluster 2 are underlined (padj<0.05), "“” indicates genes with a 1068 log2foldchange $>0.59$ or $<-0.59$, “**” indicate genes with log2foldchange $>1.0$ or $<-1.0$. 

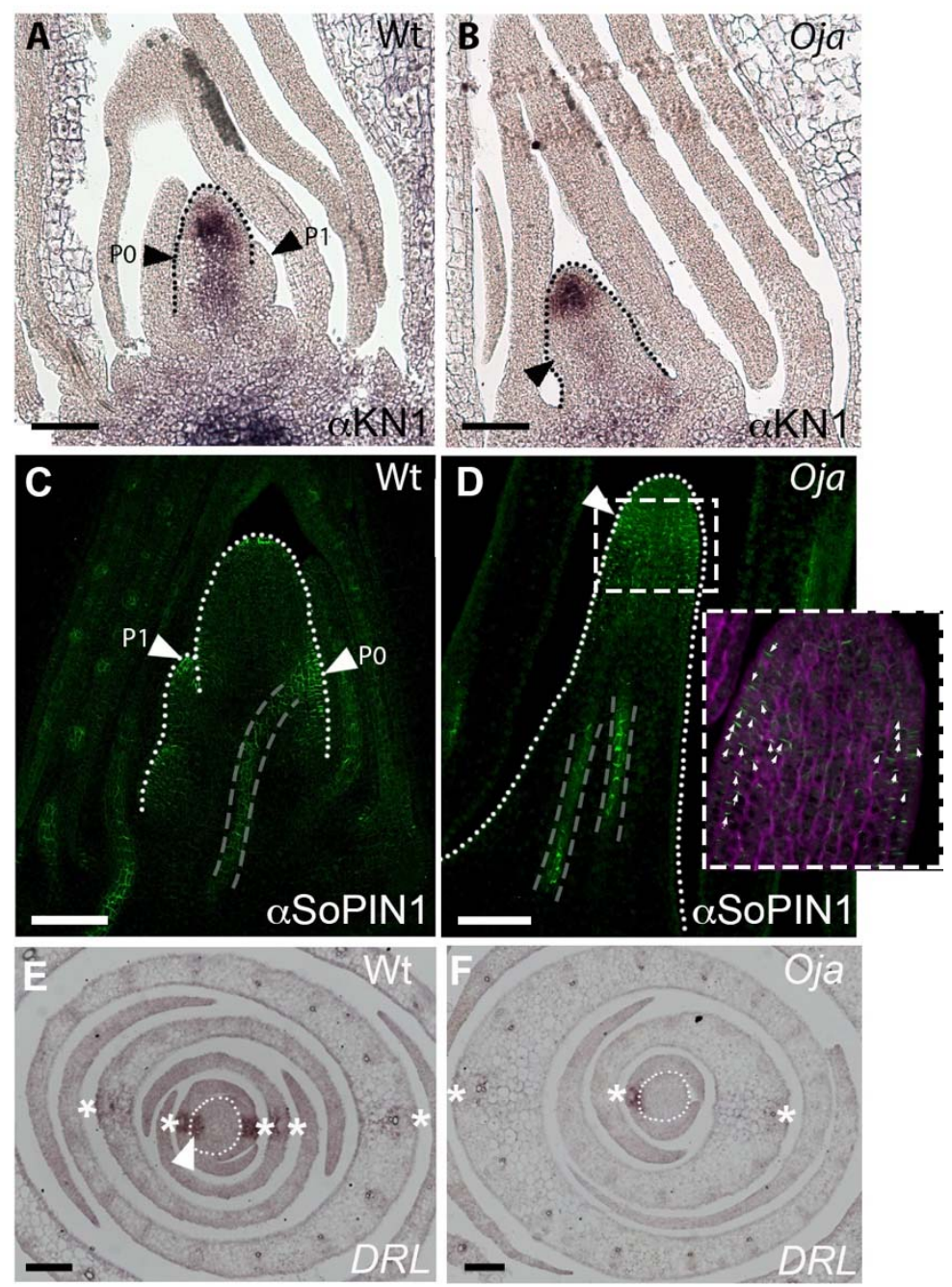

Figure 7. Hoja loca1 have defects in organ initiation markers localization. PINFORMED1 (SoPIN1, green, $C-D$ ) in longitudinal sections through 3-week-old seedlings in WT $(A, C)$ and mutant $(B, D)$ plants. The inset in $D$ shows a magnified image of the boxed region, the arrowheads indicate the orientation of the SoPIN1 localization (green) within the cell outline (magenta). Large arrowheads indicate the $P_{0}$ and $P_{1}$ domains. Dotted lines indicate the outline of the meristem region. The white dotted line in $\mathrm{A}$ indicate the $\mathrm{P}_{0}$. Grey dashed lines indicate vascular traces. (E-F) In situ hybridization of drooping leaf (dark brown, DL) in transverse cross-sections through 3week-old seedlings, in wild type (E) and mutant (F) plants. Sections are $100 \mu \mathrm{m}$ from the tip of the meristem. * indicate the position of the midvein in successive leaves, the white dotted line outlines the meristem. Scale bars are $100 \mu \mathrm{m}$. 


\begin{tabular}{|l|l|}
\hline Primer Name & Sequence (Forward/Reverse) \\
\hline AS_1 & TGGGCCATGCTAGGCATTAACT/ TCTGGCTCCTCTCTACCGGA \\
\hline AS_2 & CGTCCTCCAGCTTAGTGTTG/ CTTCGACGACTTCTCCTTCC \\
\hline AS_3 & GGGCTGGTTTGGTGGCAGGG/ TGTCATGCGTCCGTCACTGCT \\
\hline AS_4 & GGTTTCCCGGAGGAGGAATG/ AGTTAGTCGCCTCTTTCGCC \\
\hline AS_5 & ATGCCAGGAAATCACGGAGG/ GAGTGTTTCCGAGGCTTTGC \\
\hline
\end{tabular}
map the Oja mutation. Primer locations are indicated in Figure. 4. 


\section{Supplementary Figures}
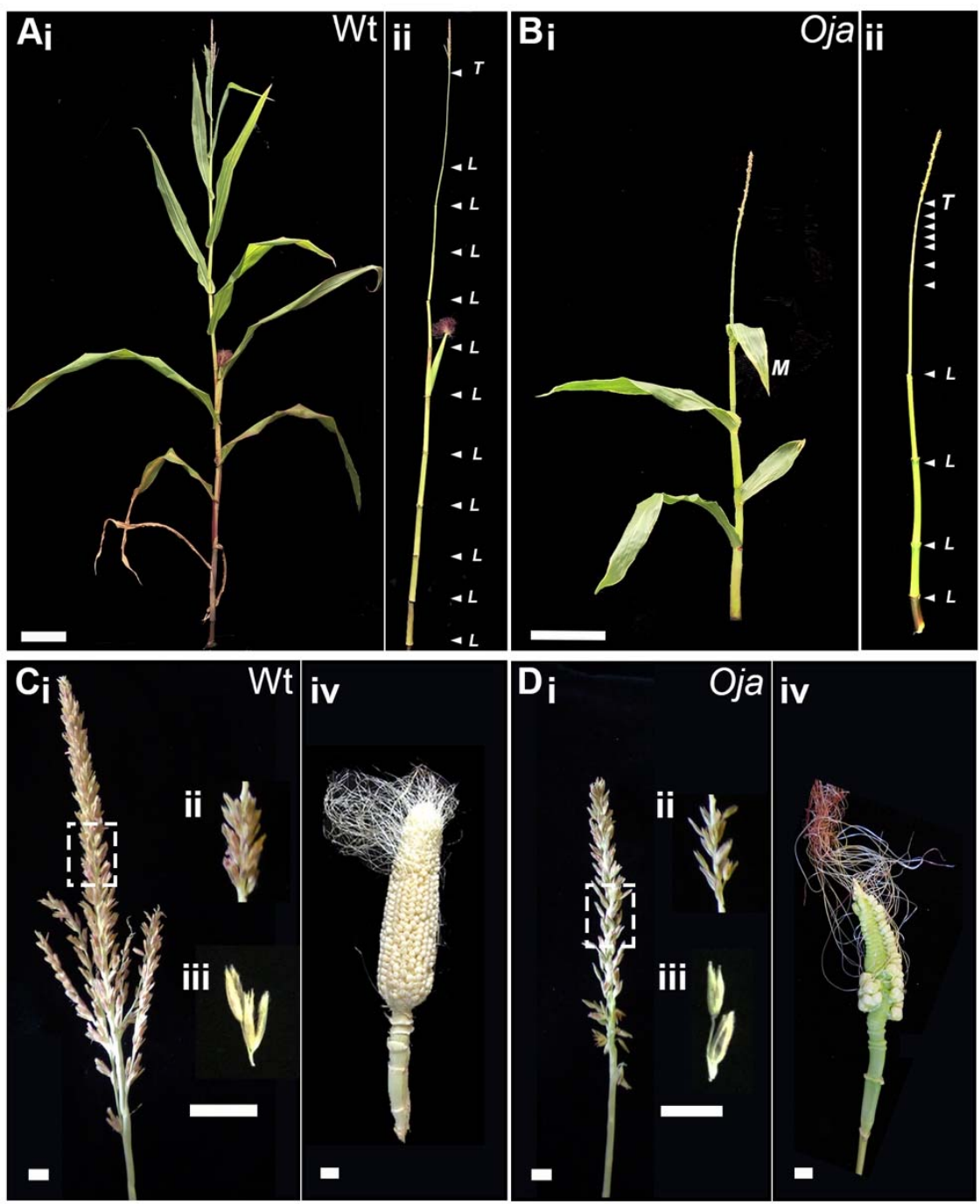

\section{Supplementary Figure 1. Hoja loca1 Mature Phenotype.}

1093 The mature phenotype of a wild type (A) and mutant (B) plant, with leaves attached (i) 1094 and removed (ii), scale bar is $15 \mathrm{~cm}$. The positions of nodes are indicated by the white 1095 arrowheads, those that have a leaf are indicated by " $\mathrm{"} \mathrm{and} \mathrm{the} \mathrm{base} \mathrm{of} \mathrm{the} \mathrm{tassel} \mathrm{is}$ indicated by "T". The phenotype of tassels and ears in wild type (C) and mutant (D) plants. (i) Whole tassel, (ii) zoomed-in image of the boxed region, (iii) an individual spikelet pair, (iv) unfertilized ear. Scale bars are $1 \mathrm{~cm}$. 

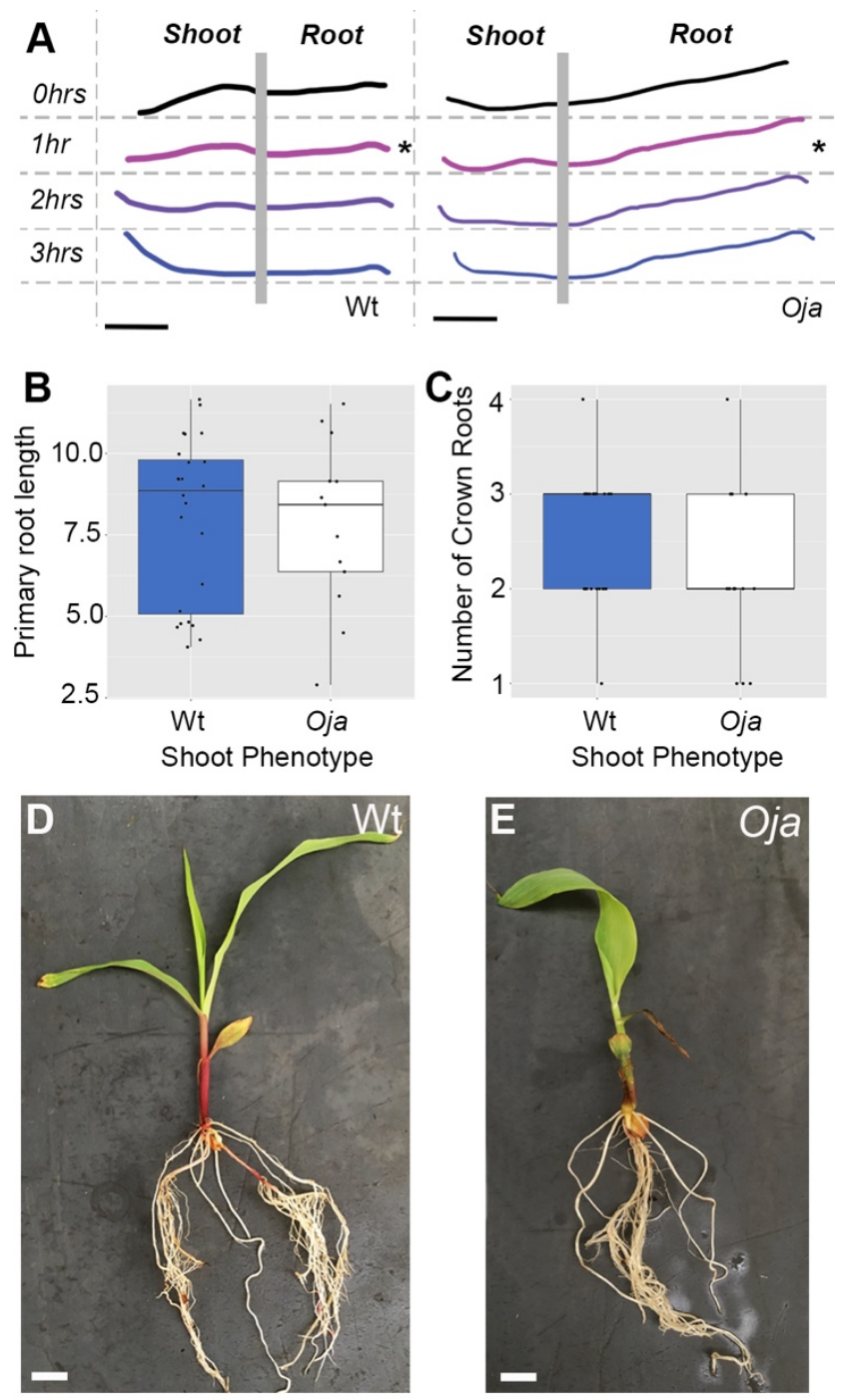

1100 Supplementary Figure 2. Hoja loca1 mutants have a normal root phenotype.

(A) Oja seedling roots have a normal gravitropic response, showing a change in root tip orientation by 1 hour after turning $\left({ }^{*}\right)$ (scale bars are $2 \mathrm{~cm}$ ). (B-C) Root architecture in 1.5-week-old seedlings is not significantly different between wild-type and Oja plants, as shown by primary root length $(B$, Welch 2 -sample $t$-test, $d f=24, n$ Oja $=13, W t=24$, $1105 \mathrm{p}=0.853)$ and number of initiating crown roots (C, Welch 2-sample $\mathrm{t}$-test, $\mathrm{df}=19, \mathrm{n}$ $O j a=13, W t=24, p=0.1452$ ). (D-E) Root architecture in 3-week-old seedlings appears to be similar between mutant and normal sibling (Wt) (scale bars are $2 \mathrm{~cm}$ ). 


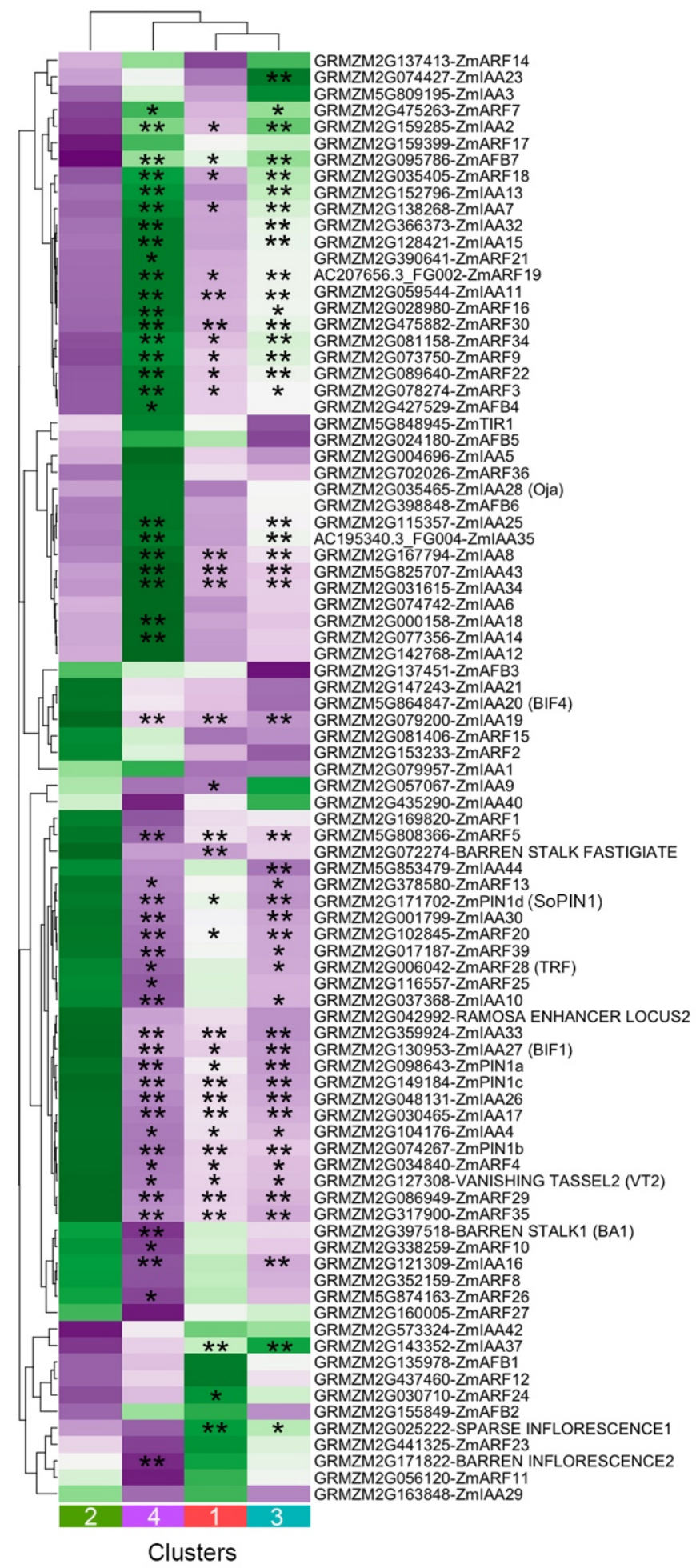

Clusters

1110 Supplementary Figure 3. Hoja loca1 mutants show changes in auxin-signaling 1111 related genes.

1112 Heatmap of average FPKM values of Auxin related genes in clusters 1-4. Colouring is 1113 relative average FPKM per row. $\left({ }^{*}{ }^{* *}\right)$ indicate genes that are significantly differentially 1114 expressed (padj<0.05), $\left(^{* *}\right)$ log2foldchange $>1$ or $<-1$, foldchange of 2 or 0.5

The author responsible for distribution of materials integral to the findings presented in this article in accordance with the policy described in the Instructions for Authors (https://academic.oup.com/plcell/pages/GeneralInstructions) is: Annis E. Richardson (annis.richardson@ed.c.uk). 
1115 respectively, $\left({ }^{*}\right)$ log2foldchange $<0.59<1$ or $>-1<0.59$, foldchange of 1.5 or 0.5 1116 respectively between the indicated cluster and cluster 2. 
Supplementary Datasets:

Supplementary Dataset 1: Broad RNAseq differential gene expression

1121 comparison between individuals scored as normal or mutant based on seedling

\section{2 phenotype.}

1123 DeSeq2 output of comparisons between samples with seedling phenotypes scored as

1124 wild-type or mutant. This data relates to the results shown in Figure 5. All significantly 1125 expressed genes between the pools of mutant and wildtype scored samples (padj $1126<0.05$ ) are listed by their gene identifiers (GID) based on the maize B73 RefGenv3 1127 genome, with matched annotations from Maize, and the best BLAST hit for Arabidopsis 1128 and rice. Negative log2FoldChange values indicate down-regulation in mutants.

1130 Supplementary Dataset 2: RNAseq differential gene expression comparison 1131 between individuals in cluster 1 versus cluster 2.

1132 DeSeq2 output of comparisons between samples defined as cluster 1 or 2 based on the 1133 hierarchical clustering. This data relates to the results shown in Figure 6. All significantly 1134 expressed genes between the cluster 1 and cluster 2 scored samples (padj <0.05) are 1135 listed by their gene identifiers (GID) based on the maize B73 RefGenv3 genome, with 1136 matched annotations from Maize, and the best BLAST hit for Arabidopsis and rice.

Supplementary Dataset 3: RNAseq differential gene expression comparison between individuals in cluster 3 versus cluster 2.

1140 DeSeq2 output of comparisons between samples defined as cluster 3 or 2 based on the 1141 hierarchical clustering. This data relates to the results shown in Figure 6. All significantly 1142 expressed genes between the cluster 3 and cluster 2 scored samples (padj <0.05) are 1143 listed by their gene identifiers (GID) based on the maize B73 RefGenv3 genome, with 1144 matched annotations from Maize, and the best BLAST hit for Arabidopsis and rice

1146 Supplementary Dataset 4: RNAseq differential gene expression comparison 1147 between individuals in cluster 4 versus cluster 2. 
1148 DeSeq2 output of comparisons between samples defined as cluster 4 or 2 based on the

1149 hierarchical clustering. This data relates to the results shown in Figure 6. All significantly

1150 expressed genes between the cluster 4 and cluster 2 scored samples (padj <0.05) are

1151 listed by their gene identifiers (GID) based on the maize B73 RefGenv3 genome, with

1152 matched annotations from Maize, and the best BLAST hit for Arabidopsis and rice

1153

1154 Supplementary Dataset 5: Self-organising map information for all expressed 1155 genes

1156 List of all expressed genes (defined as more than 5 counts in more than 3 samples) with

1157 their associated gene identifiers (GID) based on the maize B73 RefGenv3 genome, with

1158 matched annotations from Maize, and the best BLAST hit for Arabidopsis and rice, and

1159 their node defined in the 4x4 SOM analysis shown in Figure 6D.

1160 